Research Article

\title{
Classification of Upper Bound Sequences of Local Fractional Metric Dimension of Rotationally Symmetric Hexagonal Planar Networks
}

\author{
Shahbaz Ali $\mathbb{I D}^{1,2}$ Muhammad Khalid Mahmood ${ }^{1 D},{ }^{1}$ Fairouz Tchier $\left(\mathbb{D},{ }^{3}\right.$ and F. M. O. Tawfiq ${ }^{3}$ \\ ${ }^{1}$ Department of Mathematics, University of the Punjab, Lahore 54590, Pakistan \\ ${ }^{2}$ Department of Mathematics, Khwaja Fareed University of Engineering \& Information Technology, Rahim Yar Khan, Pakistan \\ ${ }^{3}$ Department of Mathematics, King Saud University, P.O. Box 22452, Riyadh 11495, Saudi Arabia
}

Correspondence should be addressed to Fairouz Tchier; ftchier@ksu.edu.sa

Received 5 December 2020; Revised 17 January 2021; Accepted 4 February 2021; Published 28 February 2021

Academic Editor: Kinkar Chandra Das

Copyright (c) 2021 Shahbaz Ali et al. This is an open access article distributed under the Creative Commons Attribution License, which permits unrestricted use, distribution, and reproduction in any medium, provided the original work is properly cited.

The term metric or distance of a graph plays a vital role in the study to check the structural properties of the networks such as complexity, modularity, centrality, accessibility, connectivity, robustness, clustering, and vulnerability. In particular, various metrics or distance-based dimensions of different kinds of networks are used to resolve the problems in different strata such as in security to find a suitable place for fixing sensors for security purposes. In the field of computer science, metric dimensions are most useful in aspects such as image processing, navigation, pattern recognition, and integer programming problem. Also, metric dimensions play a vital role in the field of chemical engineering, for example, the problem of drug discovery and the formation of different chemical compounds are resolved by means of some suitable metric dimension algorithm. In this paper, we take rotationally symmetric and hexagonal planar networks with all possible faces. We find the sequences of the local fractional metric dimensions of proposed rotationally symmetric and planar networks. Also, we discuss the boundedness of sequences of local fractional metric dimensions. Moreover, we summarize the sequences of local fractional metric dimension by means of their graphs.

\section{Introduction}

The subject graph theory has a vital role in computer science, medical, chemical, networking, image processing, navigation, pattern recognition, drug discovery, chemical formation, sensors fixing, circuit designing, and many other fields. In graph theory, the term distance or metric plays a crucial role in solving many problems, in which we want to set the minimum distance between different objects to fixed objects, for example, if we want to fix a sensor for recognition of object, then we will fix the sensor to minimum distance from each object for better result. Moreover, when we check the structural properties of the networks such as complexity, modularity, centrality, accessibility, connectivity, robustness, clustering, and vulnerability, then metric or distance is very useful.
A graph or network is linked between two sets: one of them is a set of nodes and the other one is a set of edges. The set of vertices is denoted by $V(G)$, and $E(G)$ represents a set of edges which is a subset of $V(G) \times V(G)$. The distance or metric between two vertices $x$ and $y$ is defined as the number of minimum edges between $x$ and $y$; it is denoted as $d(x, y)$. For more details and insights into the nexus of graph theory, we refer the readers to $[1-3]$.

A subset $S=\left\{s_{1}, s_{2}, \ldots, s_{t}\right\}$ of $V(G)$ is known as resolving set if $a \in V(G)$, with $k$ - tuple metric form represented as $r(x \mid S)=\left(d\left(x, s_{1}\right), d\left(x, s_{1}\right), \ldots, d\left(x, s_{k}\right)\right)$. Then, $S$ will be a resolving set with $k$ elements if $r(x \mid S) \neq r(y \mid S)$ for each pair of distinct vertices $x$ and $y$. The minimum number set $S$ is known as the basis of $G$. The cardinality of the set $S$ is called the metric dimension of $G$; it is denoted by $\beta(G)$ in $[4,5]$. 
Slatter investigated the term resolving set of connected graphs by using the notion of locating set [6-8]. Harary and Melter [9] investigated individually the aforementioned terminologies and proclaimed them as the metric dimension of a graph. A family of graphs is bounded (constant) with respect to metric dimension if each graph in this family has bounded (constant) metric dimension, respectively. The metric dimension of path and cycle has been investigated in [10]. In [4], it is shown that Peterson, anti-prism, and circulates networks have constant metric dimension. The metric dimension of wheel and Jahangir graphs was found in [11]. In [12], the authors have shown that the generalized Peterson network $P(n, 3)$ has a bounded metric dimension. Liu et al. discussed many aspects of graph theory such as indices, metric dimension domination number, and so on in [13-21].

In 2000, Chartrand et al. found the solution of integer programming problem (IPP) by means of metric dimension [4]. For a more accurate solution of integer programming problem (IPP), Currie and Oellermann introduced the concept of fractional metric dimension in [22]. Fehr et al. found an optimal solution of a certain linear programming relaxation problem by means of fractional metric dimension [23]. Liu et al. discussed many interesting results on the fractional metric dimension (FMD) in [20]. Many results of fractional metric dimension on several networks such as networks obtained from Cartesian, hierarchical, corona, lexicographic, and comb products of connected networks have been discussed in $[4,20,24,25]$. The fractional metric dimension of the generalized Jahangir network was computed by Liu et al. in [26]. The local fractional metric dimensions of planar networks were computed in [27]. In 2019, Aisyah et al. introduced the concept of local fractional metric dimension (LFMD) and computed it for the corona product of two graphs [28]. Many researchers discussed this area in $[5,6,8,29-31]$. A new family of graphs based on totient, super totient, and hyper totient numbers was proposed in [32-36].

In this paper, we choose the family of rotationally symmetric hexagonal planar networks with all possible faces and then find their sequences of local fractional metric dimension (LFMD). Moreover, we summarize sequences of local fractional metric dimensions by means of discrete graphs. This paper proceeds in the following sequence. Section 2 gives the preliminaries. In Section 3, we define the vertex and edge sets of rotationally symmetric hexagonal planar networks and their subsections; we find the sequences of these networks by introducing lemmas and theorems. In Section 4, we give the boundedness table of sequences of local fractional metric dimension (LFMD) and also plot their discrete graphs.

\section{Preliminaries}

Let $G$ be a connected graph. A pair of vertices $\{s, t\} \subseteq V(G)$ is a resolved set by a vertex $u$ if $d(s, u) \neq d(t, u)$. The set of resolving neighborhood of pair $\{s, t\}$ is defined as $\mathscr{R}=\{u \in V(G) \mid d(s, u) \neq d(t, u)\}$. Let $G$ be a connected graph of order $n$. A function $\vartheta: V(G) \longrightarrow[0,1]$ is termed as resolving function of $G$ if $\vartheta(\mathscr{R}\{s, t\}) \geq 1, \forall s, t \in V(G)$, where

$$
\vartheta(\mathscr{R}\{s, t\})=\sum_{u \in \mathscr{R}\{s, t\}} \vartheta(u)
$$

A resolving function $\vartheta^{\prime}$ of $G$ is called a minimal resolving function if there exists $u \in V(G)$ which is not a resolving function of $G$ and if any other function $\vartheta: V(G) \longrightarrow[0,1]$ with $\vartheta \leq \vartheta^{\prime}, \quad \vartheta(u) \neq \vartheta$ r $(u)$. The fractional metric dimension (FMD) of the graph $G$ is defined as $\operatorname{dim}_{f}(G)=\min \left\{\left|\vartheta^{\prime}\right|\right.$ : $\vartheta^{\prime}$ is the minimal resolvingfunction of $G$, where

$$
\left|\vartheta^{\prime}\right|=\sum_{u \in V(G)} \vartheta^{\prime}(u)
$$

The resolving function will be a local resoling function if $\vartheta^{\prime}(\mathscr{R}\{s, t\}) \geq 1$ and the fractional metric dimension becomes a local fractional metric dimension (LFMD) if we assume the pair of adjacent vertices only; it is denoted by $\mathscr{D i m}_{l f}(G)$ $[20,28]$. The LFMD of given graph in Figure 1 is $\mathscr{D} \mathbf{i} \mathbf{m}_{l f}$ $(G) \leq(12 / 7)$.

The local resolving neighborhoods are

$$
\begin{aligned}
\mathscr{R}\left\{x_{7} x_{13}\right\} & =V(G)-\left\{x_{2}, x_{5}, x_{8}, x_{12}\right\}, \\
\mathscr{R}\left\{x_{1} x_{7}\right\} & =V(G)-\left\{x_{10}, x_{14}, x_{15}, x_{16}, x_{20}, x_{21}\right\}, \\
\mathscr{R}\left\{x_{9} x_{15}\right\} & =V(G)-\left\{x_{1}, x_{4}, x_{8}, x_{10}\right\}, \\
\mathscr{R}\left\{x_{7} x_{6}\right\} & =V(G)-\left\{x_{10}, x_{16}, x_{17}, x_{18}, x_{22}, x_{23}\right\}, \\
\mathscr{R}\left\{x_{8} x_{14}\right\} & =V(G)-\left\{x_{3}, x_{6}, x_{7}, x_{9}\right\}, \\
\mathscr{R}\left\{x_{3} x_{9}\right\} & =V(G)-\left\{x_{12}, x_{16}, x_{17}, x_{18}, x_{22}, x_{23}\right\}, \\
\mathscr{R}\left\{x_{12} x_{18}\right\} & =V(G)-\left\{x_{1}, x_{4}, x_{7}, x_{11}\right\}, \\
\mathscr{R}\left\{x_{2} x_{9}\right\} & =V(G)-\left\{x_{12}, x_{13}, x_{14}, x_{18}, x_{19}, x_{24}\right\}, \\
\mathscr{R}\left\{x_{11} x_{17}\right\} & =V(G)-\left\{x_{2}, x_{6}, x_{10}, x_{12}\right\}, \\
\mathscr{R}\left\{x_{2} x_{8}\right\} & =V(G)-\left\{x_{11}, x_{15}, x_{16}, x_{17}, x_{21}, x_{22}\right\},
\end{aligned}
$$




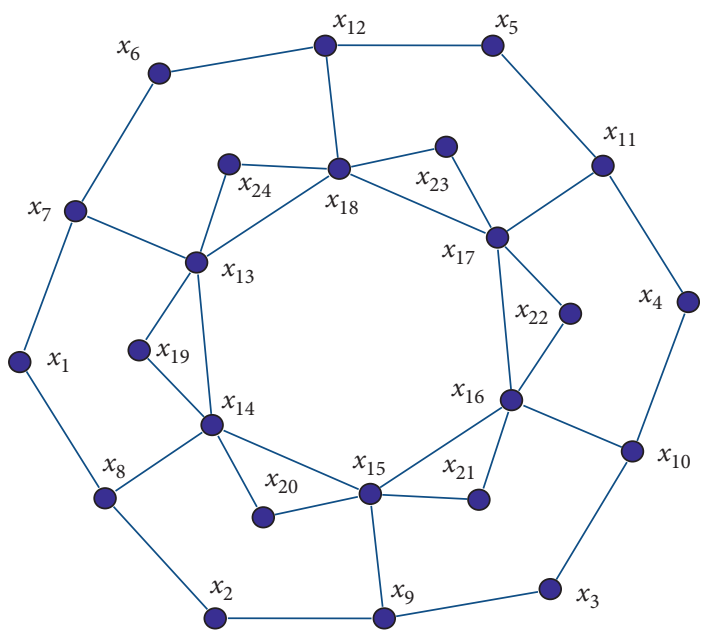

FIgURE 1: Hexagonal planar graph $G$ with $\mathscr{D i m}_{l f}(G) \leq(12 / 7)$.

$$
\begin{aligned}
\mathscr{R}\left\{x_{10} x_{16}\right\} & =V(G)-\left\{x_{2}, x_{5}, x_{9}, x_{11}\right\}, \\
\mathscr{R}\left\{x_{1} x_{8}\right\} & =V(G)-\left\{x_{11}, x_{13}, x_{17}, x_{18}, x_{23}, x_{24}\right\}, \\
\mathscr{R}\left\{x_{13} x_{18}\right\} & =V(G)-\left\{x_{3}, x_{6}, x_{21}, x_{24}\right\}, \\
\mathscr{R}\left\{x_{6} x_{12}\right\} & =V(G)-\left\{x_{9}, x_{13}, x_{14}, x_{15}, x_{19}, x_{20}\right\}, \\
\mathscr{R}\left\{x_{18} x_{17}\right\} & =V(G)-\left\{x_{2}, x_{5}, x_{20}, x_{23}\right\}, \\
\mathscr{R}\left\{x_{5} x_{12}\right\} & =V(G)-\left\{x_{9}, x_{15}, x_{16}, x_{17}, x_{21}, x_{22}\right\}, \\
\mathscr{R}\left\{x_{16} x_{17}\right\} & =V(G)-\left\{x_{1}, x_{4}, x_{9}, x_{22}\right\}, \\
\mathscr{R}\left\{x_{5} x_{11}\right\} & =V(G)-\left\{x_{8}, x_{13}, x_{14}, x_{18}, x_{19}, x_{24}\right\}, \\
\mathscr{R}\left\{x_{15} x_{16}\right\} & =V(G)-\left\{x_{3}, x_{6}, x_{21}, x_{24}\right\}, \\
\mathscr{R}\left\{x_{4} x_{11}\right\} & =V(G)-\left\{x_{8}, x_{14}, x_{15}, x_{16}, x_{20}, x_{21}\right\}, \\
\mathscr{R}\left\{x_{14} x_{15}\right\} & =V(G)-\left\{x_{2}, x_{5}, x_{20}, x_{23}\right\}, \\
\mathscr{R}\left\{x_{4} x_{10}\right\} & =V(G)-\left\{x_{7}, x_{13}, x_{17}, x_{18}, x_{23}, x_{24}\right\}, \\
\mathscr{R}\left\{x_{13} x_{14}\right\} & =V(G)-\left\{x_{1}, x_{4}, x_{19}, x_{22}\right\}, \\
\mathscr{R}\left\{x_{3} x_{10}\right\} & =V(G)-\left\{x_{7}, x_{13}, x_{14}, x_{15}, x_{19}, x_{20}\right\}, \\
\mathscr{R}\left\{x_{13} x_{24}\right\} & =\mathscr{R}\left\{x_{15} x_{21}\right\}=V(G)-\left\{x_{4}, x_{5}, x_{10}, x_{11}, x_{12}, x_{16}, x_{17}, x_{18}, x_{22}, x_{23}\right\}, \\
\mathscr{R}\left\{x_{18} x_{24}\right\} & =\mathscr{R}\left\{x_{16} x_{21}\right\}=V(G)-\left\{x_{1}, x_{2}, x_{7}, x_{8}, x_{9}, x_{13}, x_{14}, x_{15}, x_{19}, x_{20}\right\}, \\
\mathscr{R}\left\{x_{18} x_{23}\right\} & =\mathscr{R}\left\{x_{14} x_{20}\right\}=V(G)-\left\{x_{3}, x_{4}, x_{9}, x_{10}, x_{11}, x_{15}, x_{16}, x_{17}, x_{21}, x_{22}\right\}, \\
\mathscr{R}\left\{x_{17} x_{23}\right\} & =\mathscr{R}\left\{x_{15} x_{20}\right\}=V(G)-\left\{x_{1}, x_{6}, x_{7}, x_{8}, x_{12}, x_{13}, x_{14}, x_{18}, x_{19}, x_{24}\right\}, \\
\mathscr{R}\left\{x_{17} x_{22}\right\} & =\mathscr{R}\left\{x_{13} x_{19}\right\}=V(G)-\left\{x_{2}, x_{3}, x_{8}, x_{9}, x_{10}, x_{14}, x_{15}, x_{16}, x_{20}, x_{21}\right\}, \\
\mathscr{R}\left\{x_{16} x_{22}\right\} & =\mathscr{R}\left\{x_{14} x_{19}\right\}=V(G)-\left\{x_{5}, x_{6}, x_{7}, x_{11}, x_{12}, x_{13}, x_{17}, x_{18}, x_{23}, x_{24}\right\}
\end{aligned}
$$

The cardinality of each of the local resolving neighborhoods is either 20,16 , or 14 . Since 14 is less than the cardinality of all other resolving neighborhoods, we construct a mapping $\vartheta: V(G) \longrightarrow[0,1]$ as 


$$
\vartheta\left(x_{i}\right)=\vartheta\left(x_{j}\right)=\frac{1}{14}, \quad i \in\{13,16,17,18\}, j \in\{22,23,24\} .
$$

Since 14 is a minimal cardinal number, there must exist a minimal resolving function $\vartheta^{\prime}$ such that $\left|\vartheta^{\prime}\right| \leq|\vartheta|$. Hence,

$$
\mathscr{D i m}_{l f}(G) \leq \sum_{i=1}^{24} \frac{1}{14}=\frac{12}{7} \text {. }
$$

\section{Rotationally Symmetric Hexagonal Planar Networks}

In this section, we define hexagonal circular ladder networks. In Figure 2, the different hexagonal networks are given with faces.

The vertex set and edge set of hexagonal circular ladder networks are defined as

$$
\begin{aligned}
& V\left(H_{n}^{j}\right)=\left\{s_{i}, t_{i}, u_{i}, v_{i} \mid 1 \leq i \leq n\right\}, \quad j=1,2,3,4,5, \\
& E\left(H_{n}^{1}\right)=\left\{s_{i} t_{i}, t_{i} u_{i}, u_{i} v_{i}, u_{i} v_{i-1}, s_{i} t_{i+1} \mid 1 \leq i \leq n, t_{n+1}=t_{1}, v_{0}=v_{n}\right\}, \\
& E\left(H_{n}^{2}\right)=\left\{s_{i} t_{i}, t_{i} u_{i}, u_{i} v_{i}, u_{i} v_{i-1}, s_{i} t_{i+1}, s_{i} v_{i} \mid 1 \leq i \leq n, t_{n+1}=t_{1}, v_{0}=v_{n}\right\}, \\
& E\left(H_{n}^{3}\right)=\left\{s_{i} t_{i}, t_{i} u_{i}, u_{i} v_{i}, u_{i} v_{i-1}, s_{i} t_{i+1}, u_{i} u_{i+1} \mid 1 \leq i \leq n, t_{n+1}=t_{1}, u_{n+1}=u_{1}, v_{0}=v_{n}\right\}, \\
& E\left(H_{n}^{4}\right)=\left\{s_{i} t_{i}, t_{i} u_{i}, u_{i} v_{i}, u_{i} v_{i-1}, s_{i} t_{i+1}, s_{i} u_{i} \mid 1 \leq i \leq n, t_{n+1}=t_{1}, v_{0}=v_{n}\right\}, \\
& E\left(H_{n}^{5}\right)=\left\{s_{i} t_{i}, t_{i} u_{i}, u_{i} v_{i}, u_{i} v_{i-1}, s_{i} t_{i+1}, t_{i} u_{i+1} \mid 1 \leq i \leq n, t_{n+1}=t_{1}, u_{n+1}=u_{1}, v_{0}=v_{n}\right\},
\end{aligned}
$$

and hexagonal circular ladder networks $H_{n}^{i}, \quad 1 \leq i \leq 5$, are shown in Figure 3.
The vertex set and edge set of hexagonal circular ladder networks $H_{n}^{i}, \quad 6 \leq i \leq 11$, are defined as

$$
\begin{aligned}
& V\left(H_{n}^{j}\right)=\left\{s_{i}, t_{i}, u_{i}, v_{i} \mid 1 \leq i \leq n\right\}, \quad j=6,7,8,9,10,11, \\
& E\left(H_{n}^{6}\right)=\left\{s_{i} t_{i}, t_{i} u_{i}, u_{i} v_{i}, u_{i} v_{i-1}, s_{i} t_{i+1}, s_{i} v_{i}, s_{i} u_{i} \mid 1 \leq i \leq n, t_{n+1}=t_{1}, v_{0}=v_{n}\right\}, \\
& E\left(H_{n}^{7}\right)=\left\{s_{i} t_{i}, t_{i} u_{i}, u_{i} v_{i}, u_{i} v_{i-1}, s_{i} t_{i+1}, t_{i} t_{i+1}, t_{i} u_{i+1} \mid 1 \leq i \leq n, t_{n+1}=t_{1}, u_{n+1}=u_{1}, v_{0}=v_{n}\right\}, \\
& E\left(H_{n}^{8}\right)=\left\{s_{i} t_{i}, t_{i} u_{i}, u_{i} v_{i}, u_{i} v_{i-1}, s_{i} t_{i+1}, t_{i} v_{i}, t_{i} t_{i+1} \mid 1 \leq i \leq n, t_{n+1}=t_{1}, v_{0}=v_{n}\right\}, \\
& E\left(H_{n}^{9}\right)=\left\{s_{i} t_{i}, t_{i} u_{i}, u_{i} v_{i}, u_{i} v_{i-1}, s_{i} t_{i+1}, t_{i} v_{i}, t_{i} u_{i+1} \mid 1 \leq i \leq n, t_{n+1}=t_{1}, u_{n+1}=u_{1}, v_{0}=v_{n}\right\}, \\
& E\left(H_{n}^{10}\right)=\left\{s_{i} t_{i}, t_{i} u_{i}, u_{i} v_{i}, u_{i} v_{i-1}, s_{i} t_{i+1}, u_{i} u_{i+1}, t_{i} t_{i+1} \mid 1 \leq i \leq n, t_{n+1}=t_{1}, u_{n+1}=u_{1}, v_{0}=v_{n}\right\}, \\
& E\left(H_{n}^{11}\right)=\left\{s_{i} t_{i}, t_{i} u_{i}, u_{i} v_{i}, u_{i} v_{i-1}, s_{i} t_{i+1}, t_{i} v_{i}, s_{i} u_{i+1} \mid 1 \leq i \leq n, t_{n+1}=t_{1}, u_{n+1}=u_{1}, v_{0}=v_{n}\right\},
\end{aligned}
$$

and hexagonal circular ladder networks $H_{n}^{i}, \quad 6 \leq i \leq 11$, are shown in Figure 4.
The vertex set and edge set of hexagonal circular ladder networks $H_{n}^{i}, \quad 12 \leq i \leq 17$, are defined as

$$
\begin{aligned}
& V\left(H_{n}^{j}\right)=\left\{s_{i}, t_{i}, u_{i}, v_{i} \mid 1 \leq i \leq n\right\}, \quad j=12,13,14,15,16,17, \\
& E\left(H_{n}^{12}\right)=\left\{s_{i} t_{i}, t_{i} u_{i}, u_{i} v_{i}, u_{i} v_{i-1}, s_{i} t_{i+1}, s_{i} u_{i}, u_{i} u_{i+1}, s_{i} u_{i+1} \mid 1 \leq i \leq n, t_{n+1}=t_{1}, u_{n+1}=u_{1}, v_{0}=v_{n}\right\}, \\
& E\left(H_{n}^{13}\right)=\left\{s_{i} t_{i}, t_{i} u_{i}, u_{i} v_{i}, u_{i} v_{i-1}, s_{i} t_{i+1}, t_{i} t_{i+1}, u_{i} u_{i+1}, t_{i} u_{i+1} \mid 1 \leq i \leq n, t_{n+1}=t_{1}, u_{n+1}=u_{1}, v_{0}=v_{n}\right\} \\
& E\left(H_{n}^{14}\right)=\left\{s_{i} t_{i}, t_{i} u_{i}, u_{i} v_{i}, u_{i} v_{i-1}, s_{i} t_{i+1}, t_{i} v_{i}, s_{i} v_{i}, s_{i} u_{i+1} \mid 1 \leq i \leq n, t_{n+1}=t_{1}, u_{n+1}=u_{1}, v_{0}=v_{n}\right\}, \\
& E\left(H_{n}^{15}\right)=\left\{s_{i} t_{i}, t_{i} u_{i}, u_{i} v_{i}, u_{i} v_{i-1}, s_{i} t_{i+1}, t_{i} v_{i}, t_{i} u_{i+1}, s_{i} u_{i+1} \mid 1 \leq i \leq n, t_{n+1}=t_{1}, u_{n+1}=u_{1}, v_{0}=v_{n}\right\}, \\
& E\left(H_{n}^{16}\right)=\left\{s_{i} t_{i}, t_{i} u_{i}, u_{i} v_{i}, u_{i} v_{i-1}, s_{i} t_{i+1}, s_{i} u_{i}, s_{i} v_{i}, s_{i} u_{i+1} \mid 1 \leq i \leq n, t_{n+1}=t_{1}, u_{n+1}=u_{1}, v_{0}=v_{n}\right\}, \\
& E\left(H_{n}^{17}\right)=\left\{s_{i} t_{i}, t_{i} u_{i}, u_{i} v_{i}, u_{i} v_{i-1}, s_{i} t_{i+1}, t_{i} t_{i+1}, u_{i} t_{i+1}, v_{i} t_{i+1} \mid 1 \leq i \leq n, t_{n+1}=t_{1}, u_{n+1}=u_{1}, v_{0}=v_{n}\right\},
\end{aligned}
$$



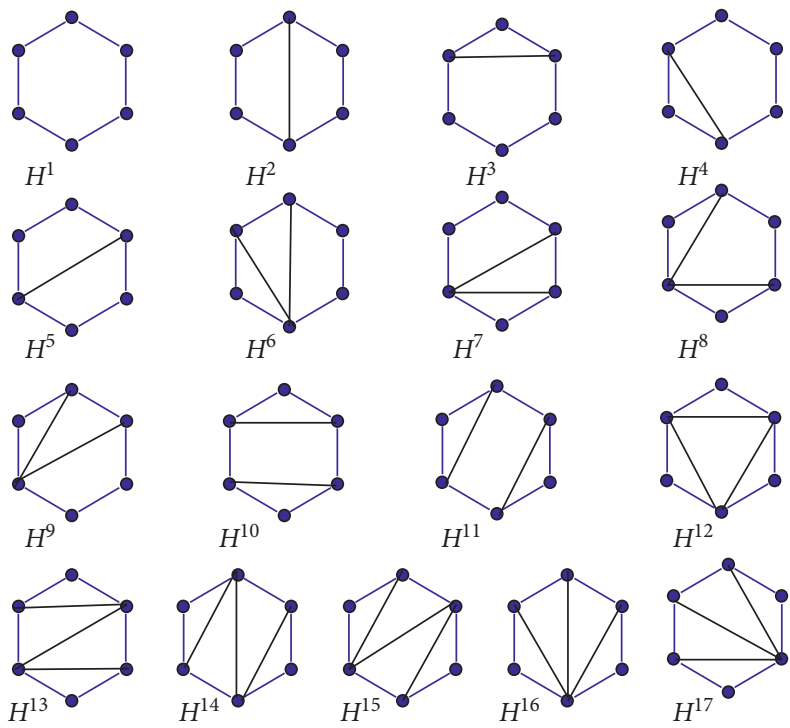

Figure 2: Hexagonal planar graphs with 2, 3, 4, and 5 faces.
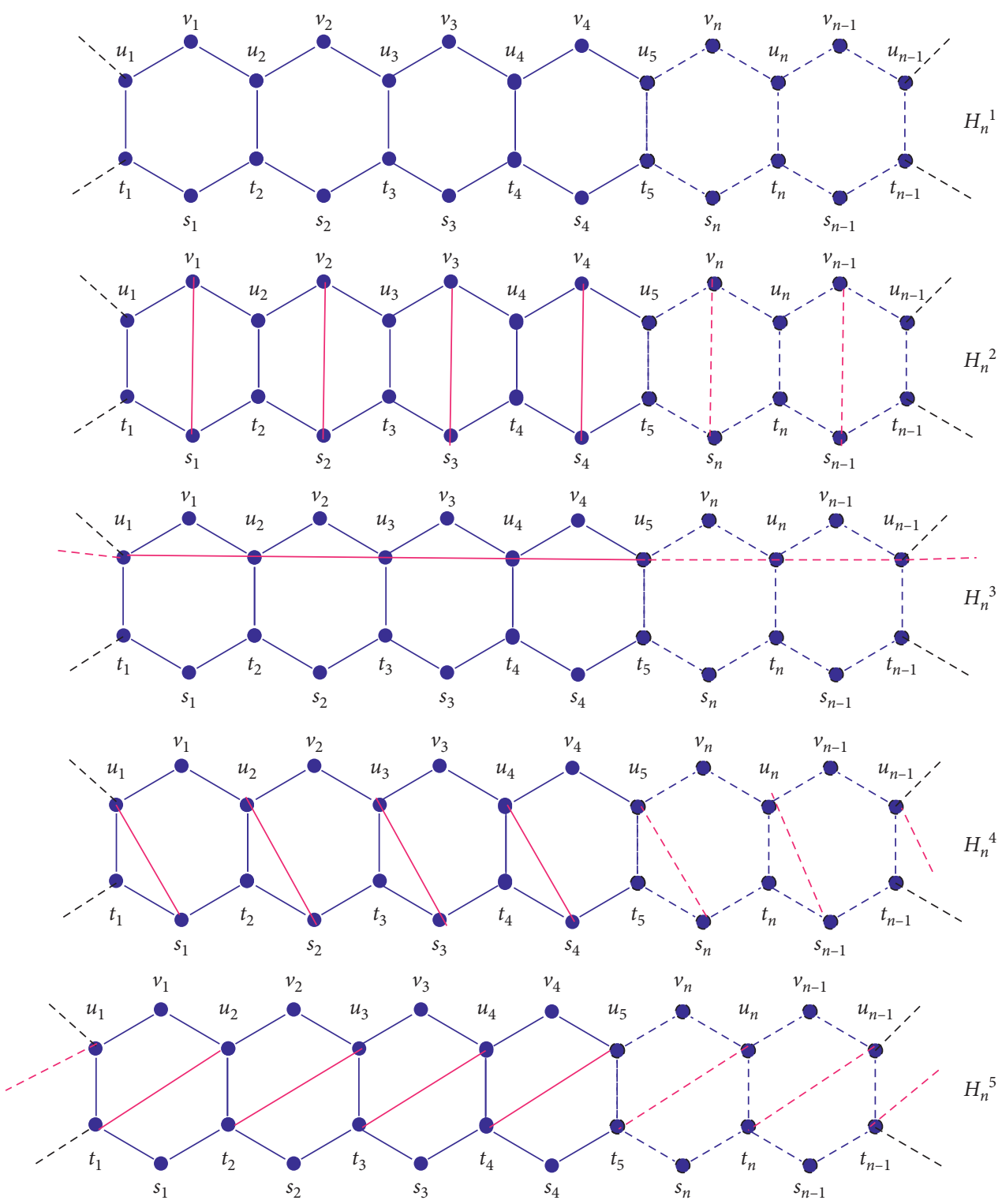

Figure 3: Rotationally symmetric hexagonal planar graphs with 2 and 3 faces. 

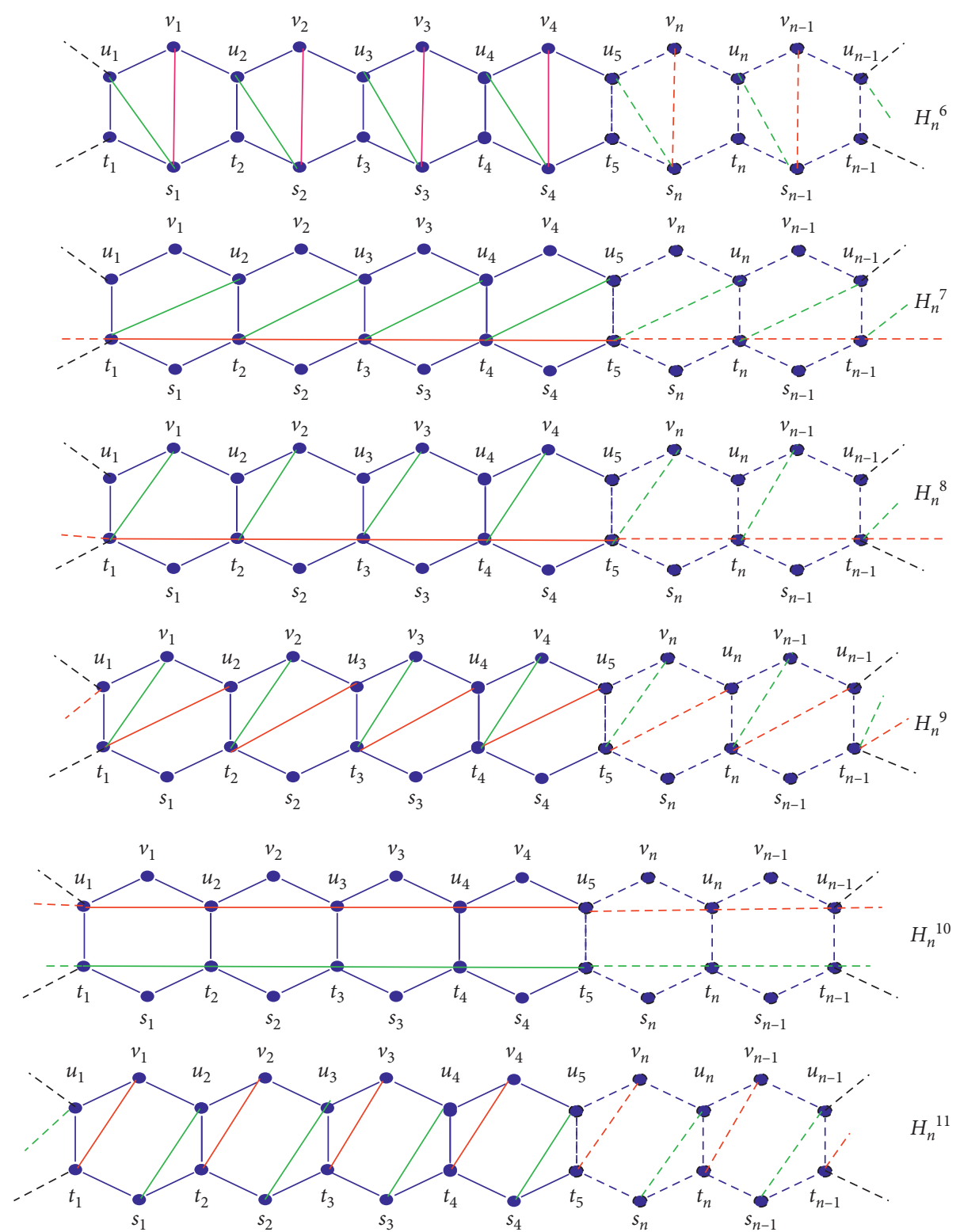

FIgURE 4: Rotationally symmetric hexagonal planar graphs with 4 faces.

and hexagonal circular ladder networks $H_{n}^{i}, \quad 12 \leq i \leq 17$, are shown in Figure 5.

3.1. LFMD of Rotationally Symmetric Hexagonal Planar Networks with 2 and 3 Faces. In this section, we find the sequences of upper bounds of local fractional metric dimension of rotationally symmetric hexagonal planar networks with 2 and 3 faces.

Javaid et. al. [37] proved that if a network is a bipartite, then its local fractional metric should be one. Since our proposed networks $H_{n}^{m}, \quad m=1,2,5$, for each $n$ are bipartite, we have the following result.

Theorem 1. The local fractional metric dimension of rotationally symmetric hexagonal planar network $H_{n}^{m}, \quad m=$ $1,2,5$, is 1. That is, $\mathscr{D i m}_{l f}\left(H_{n}^{m}\right)=1$.
Lemma 1. Let $H_{n}^{3}$ be a hexagonal planar circular network for $n \geq 5$ with $n \equiv 1(\bmod 2)$.

(1) Then, for $1 \leq k \leq n,\left|\mathscr{R}_{k}\right|=\left|\mathscr{R}\left\{u_{k} v_{k}\right\}\right|=\left|\mathscr{R}\left\{u_{k} v_{k-1}\right\}\right|$ $=2(n+1)$, with $v_{0}=v_{n}$ and $\left|\cup_{k=1}^{n} \mathscr{R}_{k}\right|=4 n$.

(2) $\left|\mathscr{R}_{k}\right|<\left|\mathscr{R}\left\{s_{k} t_{k}\right\}\right|$ and $\left|\mathscr{R}\left\{s_{k} t_{k}\right\} \cap \cup_{k=1}^{n} \mathscr{R}_{k}\right| \geq\left|\mathscr{R}_{k}\right|$.

(3) $\left|\mathscr{R}_{k}\right|<\left|\mathscr{R}\left\{s_{k} t_{k+1}\right\}\right|$ and $\left|\mathscr{R}\left\{s_{k} t_{k+1}\right\} \cap \cup_{k=1}^{n} \mathscr{R}_{k}\right| \geq\left|\mathscr{R}_{k}\right|$ with $t_{n+1}=t_{1}$.

(4) $\left|\mathscr{R}_{k}\right|<\left|\mathscr{R}\left\{t_{k} u_{k}\right\}\right|$ and $\left|\mathscr{R}\left\{t_{k} u_{k}\right\} \cap \cup_{k=1}^{n} \mathscr{R}_{k}\right| \geq\left|\mathscr{R}_{k}\right|$.

(5) $\left|\mathscr{R}_{k}\right|<\left|\mathscr{R}\left\{u_{k} u_{k+1}\right\}\right|$ and $\left|\mathscr{R}\left\{u_{k} u_{k+1}\right\} \cap \cup_{k=1}^{n} \mathscr{R}_{k}\right| \geq$ $\left|\mathscr{R}_{k}\right|$ with $u_{n+1}=u_{1}$.

Proof

(1) The set of local resolving neighborhoods of $u_{k} v_{k}$ and $u_{k} v_{k-1}$ is $\mathscr{R}\left\{u_{k} v_{k}\right\}=V\left(H_{n}^{3}\right)-\left\{s_{r} \mid r \equiv k+1, k+2\right.$, 

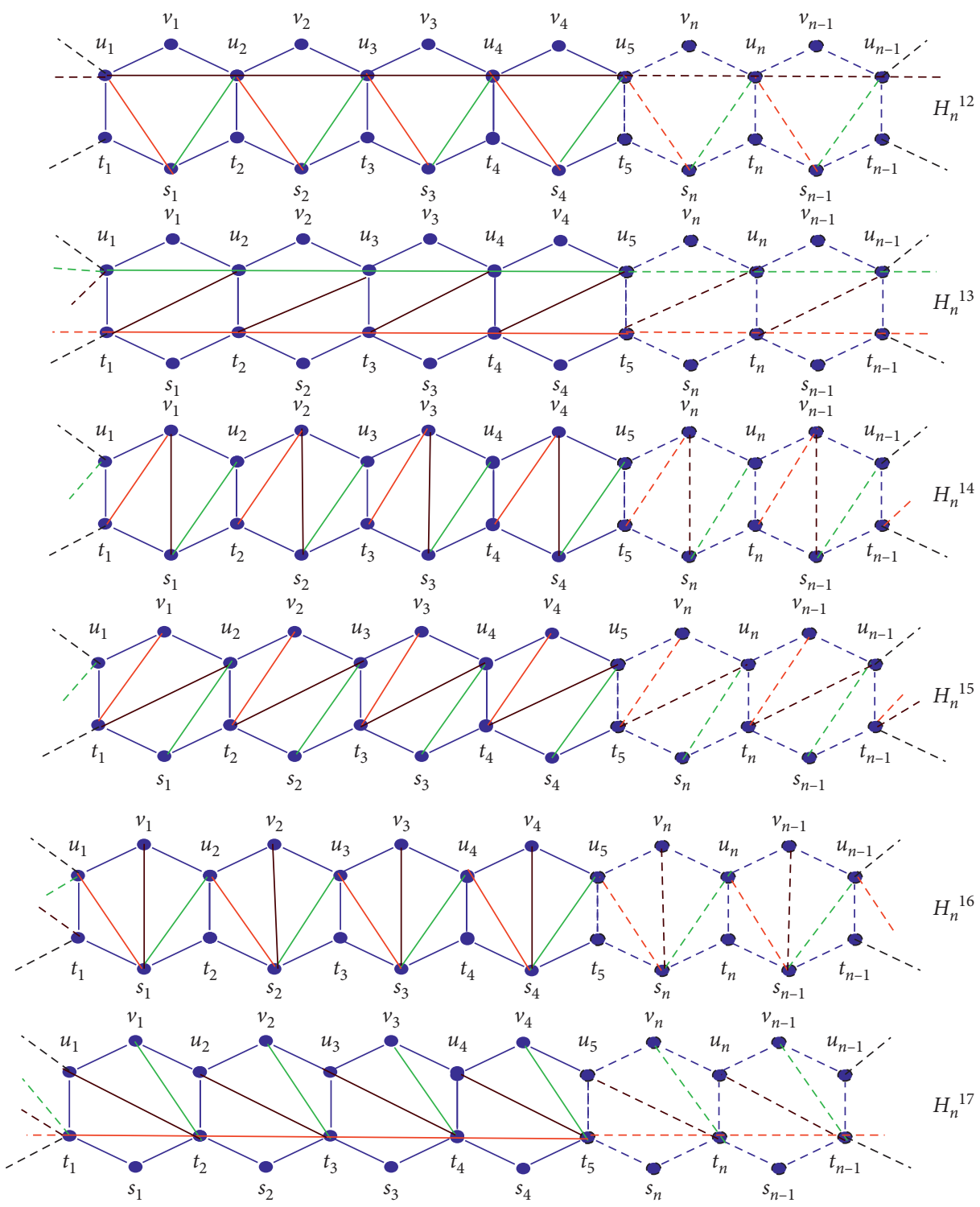

Figure 5: Rotationally symmetric hexagonal planar graphs with 5 faces.

$\ldots, k-1+(n+1 / 2)(\bmod n)\} \quad\left\{\bigcup t_{r} \mid r \equiv k+1\right.$, $k+2, \ldots, k-1+\quad(n+1 / 2)(\bmod n)\}\left\{\bigcup u_{r} \mid r\right.$ $\equiv k+1, k+2, \ldots, k-1+\quad(n+1 / 2)(\bmod n)\}$ $\left\{\bigcup v_{r} \mid r\right.$

$\equiv k+1, k+2, \ldots, k-1+(n+1 / 2)(\bmod n)\} \quad$ and $\mathscr{R}\left\{u_{k} v_{k-1}\right\}=V\left(H_{n}^{3}\right)-\left\{s_{r} \quad \mid r \equiv k+(n-1 / 2), k+1\right.$ $+(n-1 / 2), \ldots, k+n-2(\bmod n)\}\left\{\bigcup t_{r} \mid r \equiv k+1+\right.$ $(n-1 / 2), k+2+(n-1 / 2), \ldots, k+n-1(\bmod n)\}$ $\left\{\bigcup u_{r} \mid r \equiv k+1+\quad(n-1 / 2), k+2+(n-1 / \quad 2)\right.$, $\ldots, k+n-1(\bmod n)\}\left\{\bigcup v_{r} \mid r\right.$

$\equiv k+(n-1 / 2), k+1+$

$(n-1 / 2), \ldots, k+n-2(\bmod n)\} . \quad$ Clearly, $\cup_{k=1}^{n}$ $\mathscr{R}_{k}=V\left(H_{n}^{3}\right)$. Therefore, $\left|\cup_{k=1}^{n} \mathscr{R}_{k}\right|=4 n$.

(2) The set of local resolving neighborhoods of $s_{k} t_{k}$ is $\mathscr{R}\left\{s_{k} t_{k}\right\}=V\left(H_{n}^{3}\right)-\left\{s_{r} \mid r \equiv k+3, k+4, \ldots, k+(n\right.$ $+1 / 2)-1(\bmod n), n \geq 7\} \cup\left\{t_{r} \mid r \equiv k+3, k+4, \ldots, k\right.$ $+(n+1 / 2)-1(\bmod n), n \geq 7\} \cup\left\{u_{r} \mid r \equiv k+1, k+2\right.$, $\ldots, k+(n+1 / 2)-1(\bmod n), n \geq 5\} \cup\left\{v_{r} \mid r \equiv k+1\right.$, $k+2, \ldots, k+(n+1 / 2)-1(\bmod n), n \geq 5\}$. Hence, $\left|\mathscr{R}\left\{s_{k} t_{k}\right\}\right|=2 n+6$. Since $\left|\mathscr{R}_{k}\right|=2(n+1)<\mid \mathscr{R}\left\{s_{k}\right.$ $\left.t_{k}\right\}|=2 n+6,| \mathscr{R}\left\{s_{k} t_{k}\right\} \cap \cup_{k=1}^{n} \mathscr{R}_{k}|=2 n+6 \geq| \mathscr{R}_{k} \mid$.

(3) The set of local resolving neighborhoods of $s_{k} t_{k+1}$, where $t_{n+1}=t_{1}$, is $\mathscr{R}\left\{s_{k} t_{k+1}\right\}=V\left(H_{n}^{3}\right)-\left\{s_{r} \mid r \equiv k+1\right.$ $+(n-1 / 2), k+2+(n-1 / 2), \ldots, k+n-3(\bmod n), n \geq$ $7\} \cup\left\{t_{r} \mid r \equiv k+2+(n-1 / 2), k+3+(n-1 / 2), \ldots, k+n\right.$ $-2(\bmod n), n \geq 7\} \cup\left\{u_{r} \mid r \equiv k(\bmod n), r \equiv k+2+(n-\right.$ $1 / 2), k+3+(n-1 / 2), \ldots, k+n-1(\bmod n)\} \cup\left\{v_{r} \mid r \equiv\right.$ $k+1+(n-1 / 2), k+2+(n-1 / 2), \ldots, k+n-1 \quad(\bmod$ $n)\}$ Hence, $\left|\mathscr{R}\left\{s_{k} t_{k+1}\right\}\right|=2 n+6$. Since $\left|\mathscr{R}_{k}\right|=2(n+$ $1)<\left|\mathscr{R}\left\{s_{k} t_{k+1}\right\}\right|=2 n+6,\left|\mathscr{R}\left\{s_{k} t_{k+1}\right\} \cap \cup_{k=1}^{n} \mathscr{R}_{k}\right|=2 n+$ $6 \geq\left|\mathscr{R}_{k}\right|$.

(4) The set of local resolving neighborhoods of $t_{k} u_{k}$ is $\mathscr{R}\left\{t_{k} u_{k}\right\}=V\left(H_{n}^{3}\right)-\left\{s_{r} \mid r \equiv k+1(\bmod n), r \equiv k+n\right.$ $-2(\bmod n)\} \cup\left\{\begin{array}{l}t_{r} \mid r \equiv k+1(\bmod n), \\ r \equiv k+n-1(\bmod n)\end{array}\right\}$.

Hence, 
$\left|\mathscr{R}\left\{t_{k} u_{k}\right\}\right|=4(n-1)$. Since $\left|\mathscr{R}_{k}\right|=2(n+1)<\mid \mathscr{R}\left\{t_{k}\right.$ $\left.u_{k}\right\}|=4(n-1), \quad| \mathscr{R}\left\{t_{k} u_{k}\right\} \cap \cup_{k=1}^{n} \mathscr{R}_{k} \mid=4(n-1) \geq$ $\left|\mathscr{R}_{k}\right|$.

(5) The set of local resolving neighborhoods of $u_{k} u_{k+1}$, where $u_{n+1}=u_{1}$, is $\mathscr{R}\left\{u_{k} u_{k+1}\right\}=V\left(H_{n}^{3}\right)-\left\{s_{r} \mid r \equiv k\right.$ $(\bmod n)\}\left\{\bigcup t_{r} \mid r \equiv k+(n+3 / 2)-1 \quad(\bmod n)\right\} \cup\left\{u_{r}\right.$ $\mid r \equiv k+(n+3 / 2)-1(\bmod n)\}\left\{\bigcup v_{r} \mid r \equiv k(\bmod n)\right\}$. Hence, $\left|\mathscr{R}\left\{u_{k} u_{k+1}\right\}\right|=4(n-1)$. Since $\left|\mathscr{R}_{k}\right|=2$

$$
\begin{array}{lr}
(n+1)<\left|\mathscr{R}\left\{u_{k} u_{k+1}\right\}\right|=4(n-1), & \mid \mathscr{R}\left\{u_{k} u_{k+1}\right\} \cap \\
\cup_{k=1}^{n} \mathscr{R}_{k}|=4(n-1) \geq| \mathscr{R}_{k} \mid . & \square
\end{array}
$$

Theorem 2. If $\mathrm{H}_{n}^{3}$ is a hexagonal planar circular network for $n \geq 3$ with $n \equiv 1(\bmod 2)$, then $\mathscr{D i m}_{l f}\left(H_{n}^{m}\right) \leq(2 n / n+1)$.

Proof. The local resolving neighborhoods are

Case (a): for $n=3,1 \leq k \leq 3$, by Lemma 1 , we have

$$
\begin{aligned}
\overline{R_{1}}= & \mathscr{R}\left\{u_{k} v_{k}\right\}=V\left(H_{3}^{3}\right)-\left\{s_{r} \mid r \equiv k+1(\bmod 3)\right\} \cup\left\{t_{r} \mid r \equiv k+1(\bmod 3)\right\} \\
& \cup\left\{u_{r} \mid r \equiv k+1(\bmod 3)\right\} \cup\left\{v_{r} \mid r \equiv k+1(\bmod 3)\right\}, \\
\overline{R_{2}}= & \mathscr{R}\left\{u_{k} v_{k-1}\right\}=V\left(H_{3}^{3}\right)-\left\{s_{r} \mid r \equiv k+1(\bmod 3)\right\} \cup\left\{t_{r} \mid r \equiv k+2(\bmod 3)\right\} \\
& \cup\left\{u_{r} \mid r \equiv k+2(\bmod 3)\right\} \cup\left\{v_{r} \mid r \equiv k+1(\bmod 3)\right\}, \\
\overline{R_{3}}= & \mathscr{R}\left\{u_{k} u_{k+1}\right\}=V\left(H_{3}^{3}\right)-\left\{s_{r} \mid r \equiv k(\bmod 3) \cup\left\{t_{r} \mid r \equiv k+2(\bmod 3)\right\} \cup\left\{u_{r} \mid r \equiv k+2(\bmod 3)\right\}\right. \\
& \cup\left\{v_{r} \mid r \equiv k(\bmod 3)\right\}, \\
R_{1}= & \mathscr{R}\left\{s_{k} t_{k}\right\}=V\left(H_{3}^{3}\right)-\left\{u_{r} \mid r \equiv k+1(\bmod 3)\right\} \cup\left\{v_{r} \mid r \equiv k+1(\bmod 3)\right\}, \\
R_{2}= & \mathscr{R}\left\{s_{k} t_{k+1}\right\}=V\left(H_{3}^{3}\right)-\left\{u_{r} \mid r \equiv k(\bmod 3)\right\} \cup\left\{v_{r} \mid r \equiv k+1(\bmod 3)\right\}, \\
R_{3}= & \mathscr{R}\left\{t_{k} u_{k}\right\}=V\left(H_{3}^{3}\right)-\left\{s_{r} \mid r \equiv k+1(\bmod 3)\right\} \cup\left\{t_{r} \mid r \equiv k+1(\bmod 3), r \equiv k+2(\bmod 3)\right\} .
\end{aligned}
$$

The cardinality of each of the local resolving neighborhoods $\overline{R_{1}}, \overline{R_{2}}, \overline{R_{3}}, R_{1}, R_{2}$, and $R_{3}$ for each $k=1,2,3$ is $8,8,8,10,10$, and 9 , respectively. Since cardinality of $\overline{R_{1}}, \overline{R_{2}}$, and $\overline{R_{3}}$ is 8 which is less than the cardinality of all other resolving neighborhoods and $\cup_{i=1}^{3} R_{i}=V$ $\left(H_{3}^{3}\right)$, this implies that $\left|\overline{R_{j}} \cap \cup_{i=1}^{3} R_{i}\right| \geq\left|R_{i}\right|$. Now, we construct a mapping $\vartheta: V\left(H_{3}^{3}\right) \longrightarrow[0,1]$ as

$$
\vartheta\left(u_{k}\right)=\vartheta\left(v_{k-1}\right)=\vartheta\left(v_{k}\right)=\vartheta\left(u_{k+1}\right)=\frac{1}{8} \text {. }
$$

Since 8 is a very small cardinal number, there must exist a minimal resolving function $\vartheta^{\prime}$ such that $\left|\vartheta^{\prime}\right| \leq|\vartheta|$. Hence,

$$
\mathscr{D}_{\mathbf{i} \mathfrak{m}_{l f}}\left(H_{3}^{3}\right) \leq \sum_{i=1}^{12} \frac{1}{8}=\frac{3}{2}
$$

Case (b): for $n=5,1 \leq k \leq 5$, by Lemma 2, we have

$$
\begin{aligned}
\overline{R_{1}}= & \mathscr{R}\left\{u_{k} v_{k}\right\}=V\left(H_{5}^{3}\right)-\left\{s_{r} \mid r \equiv k+1, k+2(\bmod 5)\right\} \cup\left\{t_{r} \mid r \equiv k+1, k+2(\bmod 5)\right\} \cup \\
& \cdot\left\{u_{r} \mid r \equiv k+1, k+2(\bmod 5)\right\} \cup\left\{v_{r} \mid r \equiv k+1, k+2(\bmod 5)\right\}, \\
\overline{R_{2}}= & \mathscr{R}\left\{u_{k} v_{k-1}\right\}=V\left(H_{5}^{3}\right)-\left\{s_{r} \mid r \equiv k+2, k+3(\bmod 5)\right\} \cup\left\{t_{r} \mid r \equiv k+3, k+4(\bmod 5)\right\} \cup \\
& \cdot\left\{u_{r} \mid r \equiv k+3, k+4(\bmod 5)\right\} \cup\left\{v_{r} \mid r \equiv k+2, k+3(\bmod 5)\right\}, \\
R_{1}= & \mathscr{R}\left\{s_{k} t_{k}\right\}=V\left(H_{5}^{3}\right)-\left\{u_{r} \mid r \equiv k+1, k+2(\bmod 5)\right\} \cup\left\{v_{r} \mid r \equiv k+1, k+2(\bmod 5)\right\}, \\
R_{2}= & \mathscr{R}\left\{s_{k} t_{k+1}\right\}=V\left(H_{5}^{3}\right)-\left\{u_{r} \mid r \equiv k(\bmod 5), r \equiv k+4(\bmod 5)\right\} \cup\left\{v_{r} \mid r \equiv k+3, k+4(\bmod 5)\right\}, \\
R_{3}= & \mathscr{R}\left\{t_{k} u_{k}\right\}=V\left(H_{5}^{3}\right)-\left\{s_{r} \mid r \equiv k+1(\bmod 5), r \equiv k+3(\bmod 5)\right\} \cup\left\{t_{r} \mid r \equiv k+1(\bmod 5), r \equiv k+4(\bmod 5)\right\}, \\
R_{4}= & \mathscr{R}\left\{u_{k} u_{k+1}\right\}=V\left(H_{5}^{3}\right)-\left\{\begin{array}{l}
s_{r} \mid r \equiv k(\bmod 5), \cup\left\{t_{r} \mid r \equiv k+3(\bmod 5)\right\} \\
\cup\left\{u_{r} \mid r \equiv k+3(\bmod 5)\right\} \cup\left\{v_{r} \mid r \equiv k(\bmod 5)\right\} .
\end{array}\right.
\end{aligned}
$$

The cardinality of each of the local resolving neighborhoods $\overline{R_{1}}, \overline{R_{2}}, R_{1}, R_{2}, R_{3}$, and $R_{4}$ for each $1 \leq k \leq 5$ is
$12,12,16,16,16$, and 16 , respectively. Since cardinality of $\overline{R_{1}}$ and $\overline{R_{2}}$ is 12 which is less than the cardinality of all 
other resolving neighborhoods and $\cup_{i=1}^{4} R_{i}=V\left(H_{5}^{3}\right)$, this implies that $\left|\overline{R_{j}} \cap \cup_{i=1}^{4} R_{i}\right| \geq\left|R_{i}\right|$. Now, we construct a mapping $\vartheta: V\left(H_{5}^{3}\right) \longrightarrow[0,1]$ as

$$
\vartheta\left(u_{k}\right)=\vartheta\left(v_{k-1}\right)=\vartheta\left(v_{k}\right)=\frac{1}{12} \text {. }
$$

Since 12 is a very small cardinal number, there must exist a minimal resolving function $\vartheta^{\prime}$ such that $\left|\vartheta^{\prime}\right| \leq|\vartheta|$. Hence,

$$
\mathscr{D i m}_{l f}\left(H_{5}^{3}\right) \leq \sum_{i=1}^{20} \frac{1}{12}=\frac{5}{3}
$$

Case (c): for $n=7,1 \leq k \leq 7$, by Lemma 2, we have

$$
\begin{aligned}
\overline{R_{1}}= & \mathscr{R}\left\{u_{k} v_{k}\right\}=V\left(H_{7}^{3}\right)-\left\{s_{r} \mid r \equiv k+1, k+2, k+3(\bmod 7)\right\} \cup\left\{t_{r} \mid r \equiv k+1, k+2, k+3(\bmod 7)\right\} \\
& \cup\left\{u_{r} \mid r \equiv k+1, k+2, k+3(\bmod 7)\right\} \cup\left\{v_{r} \mid r \equiv k+1, k+2, k+3(\bmod 7)\right\}, \\
\overline{R_{2}}= & \mathscr{R}\left\{u_{k} v_{k-1}\right\}=V\left(H_{7}^{3}\right)-\left\{s_{r} \mid r \equiv k+3, k+4, k+5(\bmod 7)\right\} \cup\left\{t_{r} \mid r \equiv k+4, k+5, k+6(\bmod 7)\right\} \\
& \cup\left\{u_{r} \mid r \equiv k+4, k+5, k+6(\bmod 7)\right\} \cup\left\{v_{r} \mid r \equiv k+3, k+4, k+5(\bmod 7)\right\}, \\
R_{1}= & \mathscr{R}\left\{s_{k} t_{k}\right\}=V\left(H_{7}^{3}\right)-\left\{s_{r} \mid r \equiv k+3(\bmod 7)\right\} \cup\left\{t_{r} \mid r \equiv k+3(\bmod 7)\right\} \\
& \cup\left\{u_{r} \mid r \equiv k+1, k+2, k+3(\bmod 7)\right\} \cup\left\{v_{r} \mid r \equiv k+1, k+2, k+3(\bmod 7)\right\}, \\
R_{2}= & \mathscr{R}\left\{s_{k} t_{k+1}\right\}=V\left(H_{7}^{3}\right)-\left\{s_{r} \mid r \equiv k+3(\bmod 7)\right\} \cup\left\{t_{r} \mid r \equiv k+5(\bmod 7)\right\} \\
& \cup\left\{u_{r} \mid r \equiv k(\bmod 7), r \equiv k+5, k+6(\bmod 7)\right\} \cup\left\{v_{r} \mid r \equiv k+4, k+5, k+6(\bmod 7)\right\} . \\
R_{3}= & \mathscr{R}\left\{t_{k} u_{k}\right\}=V\left(H_{7}^{3}\right)-\left\{s_{r} \mid r \equiv k+1(\bmod 7), r \equiv k+5(\bmod 7)\right\} \cup \\
& \cdot\left\{t_{r} \mid r \equiv k+1(\bmod 7), r \equiv k+6(\bmod 7)\right\}, \\
R_{4}= & \mathscr{R}\left\{u_{k} u_{k+1}\right\}=V\left(H_{7}^{3}\right)-\left\{\begin{array}{c}
s_{r} \mid r \equiv k(\bmod 7), \cup\left\{t_{r} \mid r \equiv k+4(\bmod 7)\right\} \\
\left.\cup\left\{u_{r} \mid r \equiv k+4(\bmod 7)\right\} \cup\left\{v_{r} \mid r \equiv k(\bmod 7)\right\}\right\} .
\end{array}\right.
\end{aligned}
$$

The cardinality of each of the local resolving neighborhoods $\overline{R_{1}}, \overline{R_{2}}, R_{1}, R_{2}, R_{3}$, and $R_{4}$ for each $1 \leq k \leq 7$ is $16,16,20,20,24$, and 24 , respectively. Since cardinality of $\overline{R_{1}}$ and $\overline{R_{2}}$ is 16 which is less than the cardinality of all other resolving neighborhoods and $\cup_{i=1}^{4} R_{i}=V\left(H_{7}^{3}\right)$, this implies that $\left|\overline{R_{j}} \cap \cup_{i=1}^{4} R_{i}\right| \geq\left|R_{i}\right|$. Now, we construct a mapping $9: V\left(H_{7}^{3}\right) \longrightarrow[0,1]$ as

$$
\vartheta\left(u_{k}\right)=\vartheta\left(v_{k-1}\right)=\vartheta\left(v_{k}\right)=\frac{1}{16} \text {. }
$$

Since 16 is a very small cardinal number, there must exist a minimal resolving function $\vartheta^{\prime}$ such that $\left|\vartheta^{\prime}\right| \leq|\vartheta|$. Hence,

$$
\mathscr{D i m}_{l f}\left(H_{7}^{3}\right) \leq \sum_{i=1}^{28} \frac{1}{16}=\frac{7}{4}
$$

$$
\begin{aligned}
\overline{R_{1}}= & \mathscr{R}\left\{u_{k} v_{k}\right\}=V\left(H_{n}^{3}\right)-\left\{s_{r} \mid r \equiv k+1, k+2, \ldots, k-1+\frac{n+1}{2}(\bmod n)\right\} \cup \\
& \cdot\left\{t_{r} \mid r \equiv k+1, k+2, \ldots, k-1+\frac{n+1}{2}(\bmod n)\right\} \cup\left\{u_{r} \mid r \equiv k+1, k+2, \ldots, k-1+\frac{n+1}{2}(\bmod n)\right\} \\
& \cup\left\{v_{r} \mid r \equiv k+1, k+2, \ldots, k-1+\frac{n+1}{2}(\bmod n)\right\}, \\
\overline{R_{2}}= & \mathscr{R}\left\{u_{k} v_{k-1}\right\}=V\left(H_{n}^{3}\right)-\left\{s_{r} \mid r \equiv k+\frac{n-1}{2}, k+1+\frac{n-1}{2}, \ldots, k+n-2(\bmod n)\right\} \cup \\
& \cdot\left\{t_{r} \mid r \equiv k+1+\frac{n-1}{2}, k+2+\frac{n-1}{2}, \ldots, k+n-1(\bmod n)\right\} \cup
\end{aligned}
$$




$$
\begin{aligned}
& \cdot\left\{u_{r} \mid r \equiv k+1+\frac{n-1}{2}, k+2+\frac{n-1}{2}, \ldots, k+n-1(\bmod n)\right\} \cup \\
& \cdot\left\{v_{r} \mid r \equiv k+\frac{n-1}{2}, k+1+\frac{n-1}{2}, \ldots, k+n-2(\bmod n)\right\} \\
& R_{1}=\mathscr{R}\left\{s_{k} t_{k}\right\}=V\left(H_{n}^{3}\right)-\left\{s_{r} \mid r \equiv k+3, k+4, \ldots, k+\frac{n+1}{2}-1(\bmod n), n \geq 7\right\} \cup \\
& \cdot\left\{t_{r} \mid r \equiv k+3, k+4, \ldots, k+\frac{n+1}{2}-1(\bmod n), n \geq 7\right\} \cup \\
& \cdot\left\{u_{r} \mid r \equiv k+1, k+2, \ldots, k+\frac{n+1}{2}-1(\bmod n), n \geq 5\right\} \cup \\
& \left\{v_{r} \mid r \equiv k+1, k+2, \ldots, k+\frac{n+1}{2}-1(\bmod n), n \geq 5\right\} \text {, } \\
& R_{2}=\mathscr{R}\left\{s_{k} t_{k+1}\right\}=V\left(H_{n}^{3}\right)-\left\{s_{r} \mid r \equiv k+1+\frac{n-1}{2}, k+2+\frac{n-1}{2}, \ldots, k+n-3(\bmod n), n \geq 7\right\} \cup \\
& \cdot\left\{t_{r} \mid r \equiv k+2+\frac{n-1}{2}, k+3+\frac{n-1}{2}, \ldots, k+n-2(\bmod n), n \geq 7\right\} \cup \\
& \cdot\left\{u_{r} \mid r \equiv k(\bmod n), r \equiv k+2+\frac{n-1}{2}, k+3+\frac{n-1}{2}, \ldots, k+n-1(\bmod n)\right\} \cup \\
& \left\{v_{r} \mid r \equiv k+1+\frac{n-1}{2}, k+2+\frac{n-1}{2}, \ldots, k+n-1(\bmod n)\right\}, \\
& R_{3}=\mathscr{R}\left\{t_{k} u_{k}\right\}=V\left(H_{n}^{3}\right)-\left\{s_{r} \mid r \equiv k+1(\bmod n), r \equiv k+n-2(\bmod n)\right\} \cup \\
& \cdot\left\{t_{r} \mid r \equiv k+1(\bmod n), r \equiv k+n-1(\bmod n)\right\}, \\
& R_{4}=\mathscr{R}\left\{u_{k} u_{k+1}\right\}=V\left(H_{n}^{3}\right)-\left\{s_{r} \mid r \equiv k(\bmod n)\right\} \cup\left\{t_{r} \mid r \equiv k+\frac{n+3}{2}-1(\bmod n)\right\} \cup \\
& \cdot\left\{u_{r} \mid r \equiv k+\frac{n+3}{2}-1(\bmod n)\right\}\left\{\bigcup v_{r} \mid r \equiv k(\bmod n)\right\}
\end{aligned}
$$

The cardinality of each of the local resolving neighborhoods $\overline{R_{1}}, \overline{R_{2}}, R_{1}, R_{2}, R_{3}$, and $R_{4}$ for each $1 \leq k \leq n$ is $2(n+1), 2(n+1), 2 n+6,2 n+6,4(n-1)$, and $4(n-1)$, respectively. Since cardinality of $\overline{R_{1}}$ and $\overline{R_{2}}$ is $2(n+1)$ which is less than the cardinality of all other resolving neighborhoods and $\cup_{i=1}^{4} R_{i}=V\left(H_{n}^{3}\right)$, this implies that $\left|\overline{R_{j}} \cap \cup_{i=1}^{4} R_{i}\right| \geq\left|R_{i}\right|$. Now, we construct a mapping ७: $V\left(H_{n}^{3}\right) \longrightarrow[0,1]$ as

$$
\vartheta\left(u_{k}\right)=\vartheta\left(v_{k-1}\right)=\vartheta\left(v_{k}\right)=\frac{1}{2(n+1)} .
$$

Since $2(n+1)$ is a very small cardinal number, there must exist a minimal resolving function $\vartheta^{\prime}$ such that $\left|\vartheta^{\prime}\right| \leq|\vartheta|$. Hence,

$$
\mathscr{D i m}_{l f}\left(H_{n}^{3}\right) \leq \sum_{i=1}^{4 n} \frac{1}{2(n+1)}=\frac{2 n}{n+1} .
$$

Lemma 2. Let $H_{n}^{4}$ be a hexagonal planar circular network for $n \geq 3$ with $n \equiv 1(\bmod 2)$.
(1) Then, for $1 \leq k \leq n,\left|\mathscr{R}_{k}\right|=\left|\mathscr{R}\left\{t_{k} u_{k}\right\}\right|=\left|\mathscr{R}\left\{s_{k} u_{k}\right\}\right|=$ $2 n+1$, and $\left|\cup_{k=1}^{n} \mathscr{R}_{k}\right|=4 n$.

(2) $\left|\mathscr{R}_{k}\right|<\left|\mathscr{R}\left\{s_{k} t_{k}\right\}\right|$ and $\left|\mathscr{R}\left\{s_{k} t_{k}\right\} \cap \cup_{k=1}^{n} \mathscr{R}_{k}\right| \geq\left|\mathscr{R}_{k}\right|$.

(3) $\left|\mathscr{R}_{k}\right|<\left|\mathscr{R}\left\{s_{k} t_{k+1}\right\}\right|$ and $\left|\mathscr{R}\left\{s_{k} t_{k+1}\right\} \cap \cup_{k=1}^{n} \mathscr{R}_{k}\right| \geq\left|\mathscr{R}_{k}\right|$ with $t_{n+1}=t_{1}$.

(4) $\left|\mathscr{R}_{k}\right|<\left|\mathscr{R}\left\{u_{k} v_{k}\right\}\right|$ and $\left|\mathscr{R}\left\{u_{k} v_{k}\right\} \cap \cup_{k=1}^{n} \mathscr{R}_{k}\right| \geq\left|\mathscr{R}_{k}\right|$.

(5) $\left|\mathscr{R}_{k}\right|<\left|\mathscr{R}\left\{u_{k} v_{k-1}\right\}\right|$ and $\left|\mathscr{R}\left\{u_{k} v_{k-1}\right\} \cap \cup_{k=1}^{n} \mathscr{R}_{k}\right| \geq$ $\left|\mathscr{R}_{k}\right|$ with $v_{0}=v_{n}$.

Theorem 3. If $H_{n}^{4}$ is a hexagonal planar circular network for $n \geq 3$ with $n \equiv 1(\bmod 2)$, then $\mathscr{D i m}_{l f}\left(H_{n}^{m}\right) \leq(4 n / 2 n+1)$.

3.2. LFMD of Rotationally Symmetric Hexagonal Planar Networks with 4 Faces. In this section, we find the sequences of upper bounds of local fractional metric dimension of rotationally symmetric hexagonal planar networks with 4 faces.

Lemma 3. Let $H_{n}^{6}$ be a hexagonal planar circular network for $n \geq 3$ with $n \equiv 1(\bmod 2)$. 
(1) Then, for $1 \leq k \leq n, \quad\left|\mathscr{R}_{k}\right|=\left|\mathscr{R}\left\{s_{k} u_{k}\right\}\right|=2 n$, and $\left|\cup_{k=1}^{n} \mathscr{R}_{k}\right|=4 n$.

(2) $\left|\mathscr{R}_{k}\right|<\left|\mathscr{R}\left\{s_{k} t_{k}\right\}\right|$ and $\left|\mathscr{R}\left\{s_{k} t_{k}\right\} \cap \cup \cup_{k=1}^{n} \mathscr{R}_{k}\right| \geq\left|\mathscr{R}_{k}\right|$.

(3) $\left|\mathscr{R}_{k}\right|<\left|\mathscr{R}\left\{s_{k} t_{k+1}\right\}\right|$ and $\left|\mathscr{R}\left\{s_{k} t_{k+1}\right\} \cap \cup_{k=1}^{n} \mathscr{R}_{k}\right| \geq\left|\mathscr{R}_{k}\right|$ with $t_{n+1}=t_{1}$.

(4) $\left|\mathscr{R}_{k}\right|<\left|\mathscr{R}\left\{u_{k} v_{k}\right\}\right|$ and $\left|\mathscr{R}\left\{u_{k} v_{k}\right\} \cap \cup_{k=1}^{n} \mathscr{R}_{k}\right| \geq\left|\mathscr{R}_{k}\right|$.

(5) $\left|\mathscr{R}_{k}\right|<\left|\mathscr{R}\left\{u_{k} v_{k-1}\right\}\right|$ and $\left|\mathscr{R}\left\{u_{k} v_{k-1}\right\} \cap \cup_{k=1}^{n} \mathscr{R}_{k}\right|$ $\geq\left|\mathscr{R}_{k}\right|$ with $v_{0}=v_{n}$.

(6) $\left|\mathscr{R}_{k}\right|<\left|\mathscr{R}\left\{t_{k} u_{k}\right\}\right|$ and $\left|\mathscr{R}\left\{t_{k} u_{k}\right\} \cap \cup_{k=1}^{n} \mathscr{R}_{k}\right| \geq\left|\mathscr{R}_{k}\right|$.

(7) $\left|\mathscr{R}_{k}\right|<\left|\mathscr{R}\left\{s_{k} v_{k}\right\}\right|$ and $\left|\mathscr{R}\left\{s_{k} v_{k}\right\} \cap \cup_{k=1}^{n} \mathscr{R}_{k}\right| \geq\left|\mathscr{R}_{k}\right|$.

Proof

(1) The set of local resolving neighborhoods of $s_{k} u_{k}$ is $\mathscr{R}\left\{s_{k} u_{k}\right\}=V\left(H_{n}^{6}\right)-\left\{s_{r} \mid r \equiv k+1+(n-1 / 2), k+2+(n\right.$ $-1 / 2), \ldots, k+n-1(\bmod n)\}\left\{\bigcup t_{r} \mid r \equiv k(\bmod n), r \equiv k\right.$ $+1+(n-1 / 2), k+2+(n-1 / 2), \ldots, k+n-1(\bmod n)\}$ $\left\{\bigcup u_{r} \mid r \equiv k+1, k+2, \ldots, k+(n-1 / 2)(\bmod n)\right\}\left\{\bigcup v_{r} \mid\right.$ $r \equiv k, k+1, \ldots, k-1+(n+1 / 2)(\bmod n)\} . \quad$ Clearly, $\cup_{k=1}^{n} \mathscr{R}_{k}=V\left(H_{n}^{6}\right)$. Therefore, $\left|\cup_{k=1}^{n} \mathscr{R}_{k}\right|=4 n$.

(2) The set of local resolving neighborhoods of $s_{k} t_{k}$ is $\mathscr{R}\left\{s_{k} t_{k}\right\}=V\left(H_{n}^{6}\right)-\left\{u_{r} \mid r \equiv k(\bmod n)\right\} \cup\left\{v_{r} \mid r \equiv k\right.$ $-(n-1 / 2)(\bmod n), r \equiv k+n-1(\bmod n)\}$. Hence, $\left|\mathscr{R}\left\{s_{k} t_{k}\right\}\right|=4 n-3$. Since $\left|\mathscr{R}_{k}\right|=2 n<\left|\mathscr{R}\left\{s_{k} t_{k}\right\}\right|$ $=4 n-3,\left|\mathscr{R}\left\{s_{k} t_{k}\right\} \cap \cup_{k=1}^{n} \mathscr{R}_{k}\right|=4 n-3 \geq\left|\mathscr{R}_{k}\right|$.

(3) The set of local resolving neighborhoods of $s_{k} t_{k+1}$ with $t_{k+1}=t_{1}$ is $\mathscr{R}\left\{s_{k} t_{k+1}\right\}=V\left(H_{n}^{6}\right)-\left\{u_{r} \mid r \equiv k+\right.$ $(n-1 / 2)+1(\bmod n)\}$. Hence, $\left|\mathscr{R}\left\{s_{k} t_{k+1}\right\}\right|=4 n-1$. Since $\quad\left|\mathscr{R}_{k}\right|=2 n<\left|\mathscr{R}\left\{s_{k} t_{k+1}\right\}\right|=4 n-1$, $\left|\mathscr{R}\left\{s_{k} t_{k+1}\right\} \cap \cup_{k=1}^{n} \mathscr{R}_{k}\right|=4 n-1 \geq\left|\mathscr{R}_{k}\right|$.
(4) The set of local resolving neighborhoods of $u_{k} v_{k}$ is $\mathscr{R}\left\{u_{k} v_{k}\right\}=V\left(H_{n}^{6}\right)-\left\{s_{r} \mid r \equiv k(\bmod n)\right\} \cup\left\{t_{r} \mid r \equiv k\right.$ $+1(\bmod n), r \equiv k+(n+1 / 2)(\bmod n)\}$. Hence, $\mid \mathscr{R}$ $\left\{u_{k} v_{k}\right\} \mid=4 n-3$. Since $\left|\mathscr{R}_{k}\right|=2 n<\left|\mathscr{R}\left\{u_{k} v_{k}\right\}\right|=$ $4 n-3,\left|\mathscr{R}\left\{u_{k} v_{k}\right\} \cap \cup_{k=1}^{n} \mathscr{R}_{k}\right|=4 n-3 \geq\left|\mathscr{R}_{k}\right|$.

(5) The set of local resolving neighborhoods of $u_{k} v_{k-1}$ with $v_{n}=v_{0}$ is $\mathscr{R}\left\{u_{k} v_{k-1}\right\}=V\left(H_{n}^{6}\right)-\left\{s_{r} \mid r \equiv k+\right.$ $(n-1 / 2)(\bmod n)\}$. Hence, $\left|\mathscr{R}\left\{u_{k} v_{k-1}\right\}\right|=4 n-1$. Since $\quad\left|\mathscr{R}_{k}\right|=2 n<\left|\mathscr{R}\left\{u_{k} v_{k-1}\right\}\right|=4 n-1$, $\left|\mathscr{R}\left\{u_{k} v_{k-1}\right\} \cap \cup_{k=1}^{n} \mathscr{R}_{k}\right|=4 n-1 \geq\left|\mathscr{R}_{k}\right|$.

(6) The set of local resolving neighborhoods of $t_{k} u_{k}$ is $\mathscr{R}\left\{t_{k} u_{k}\right\}=V\left(H_{n}^{6}\right)-\left\{s_{r} \mid r \equiv k, k+1, \ldots, k-1+(n+1 / 2)\right.$ $(\bmod n)\} \cup\left\{t_{r} \mid r \equiv k+1, k+2, \ldots, k+(n-1 / 2) \quad(\bmod \right.$ $n)\} \cup\left\{u_{r} \mid r \equiv k+1+(n-1 / 2), k+2+(n-1 / 2), \ldots, k+n\right.$ $-1(\bmod n)\} \cup\left\{v_{r} \mid r \equiv k+(n-1 / 2), k+1+(n-1 / 2), \ldots\right.$, $k+n-2(\bmod n)\}$. Hence, $\left|\mathscr{R}\left\{t_{k} u_{k}\right\}\right|=2 n+1$. Since $\left|\mathscr{R}_{k}\right|=2 n<\left|\mathscr{R}\left\{t_{k} u_{k}\right\}\right|=2 n+1,\left|\mathscr{R}\left\{t_{k} u_{k}\right\} \cap \cup_{k=1}^{n} \mathscr{R}_{k}\right|=$ $2 n+1 \geq\left|\mathscr{R}_{k}\right|$.

(7) The set of local resolving neighborhoods of $s_{k} v_{k}$ is $\mathscr{R}\left\{s_{k} v_{k}\right\}=V\left(H_{n}^{6}\right)-\left\{s_{r} \mid r \equiv k+1, k+2, \ldots, k+(n-1 / 2)\right.$ $(\bmod n)\} \cup\left\{t_{r} \mid r \equiv k+2, k+3, \ldots, k+(n+1 / 2) \quad(\bmod n)\right\}$ $\cup\left\{v_{r} \mid r \equiv k+1+(n-1 / 2), k+2+(n-1 / 2), \ldots, k+n-1\right.$ $(\bmod n)\} \cup\left\{u_{r} \mid r \equiv k(\bmod n), r \equiv k+1+(n-1 / 2), k+2+\right.$ $(n-1 / 2), \ldots, k+n-1(\bmod n)\}$. Hence, $\left|\mathscr{R}\left\{s_{k} v_{k}\right\}\right|=$ $2 n+1$. Since $\left|\mathscr{R}_{k}\right|=2 n<\left|\mathscr{R}\left\{s_{k} v_{k}\right\}\right|=2 n+1, \mid \mathscr{R}\left\{s_{k} v_{k}\right\}$ $\cap \cup_{k=1}^{n} \mathscr{R}_{k}|=2 n+1 \geq| \mathscr{R}_{k} \mid$.

Theorem 4. If $H_{n}^{6}$ is a hexagonal planar circular network for $n \geq 3$ with $n \equiv 1(\bmod 2)$, then $\mathscr{D i m}_{l f}\left(H_{n}^{6}\right) \leq 2$.

Proof. When $n \geq 3$ with $n \equiv 1(\bmod 2)$, then by Lemma 3 , the local resolving neighborhoods are

$$
\begin{aligned}
\overline{R_{1}}= & \mathscr{R}\left\{s_{k} u_{k}\right\}=V\left(H_{n}^{6}\right)-\left\{s_{r} \mid r \equiv k+1+\frac{n-1}{2}, k+2+\frac{n-1}{2}, \ldots, k+n-1(\bmod n)\right\} \cup \\
& \cdot\left\{t_{r} \mid r \equiv k(\bmod n), r \equiv k+1+\frac{n-1}{2}, k+2+\frac{n-1}{2}, \ldots, k+n-1(\bmod n)\right\} \cup \\
& \cdot\left\{u_{r} \mid r \equiv k+1, k+2, \ldots, k+\frac{n-1}{2}(\bmod n)\right\} \cup\left\{v_{r} \mid r \equiv k, k+1, \ldots, k-1+\frac{n+1}{2}(\bmod n)\right\}, \\
R_{1}= & \mathscr{R}\left\{s_{k} t_{k}\right\}=V\left(H_{n}^{6}\right)-\left\{u_{r} \mid r \equiv k(\bmod n)\right\} \cup\left\{v_{r} \mid r \equiv k-\frac{n-1}{2}(\bmod n), r \equiv k+n-1(\bmod n)\right\}, \\
R_{2}= & \mathscr{R}\left\{s_{k} t_{k+1}\right\}=V\left(H_{n}^{6}\right)-\left\{u_{r} \mid r \equiv k+\frac{n-1}{2}+1(\bmod n)\right\}, \\
R_{3}= & \mathscr{R}\left\{u_{k} v_{k}\right\}=V\left(H_{n}^{6}\right)-\left\{s_{r} \mid r \equiv k(\bmod n)\right\} \cup\left\{t_{r} \mid r \equiv k+1(\bmod n), r \equiv k+\frac{n+1}{2}(\bmod n)\right\}, \\
R_{4}= & \mathscr{R}\left\{u_{k} v_{k-1}\right\}=V\left(H_{n}^{6}\right)-\left\{s_{r} \mid r \equiv k+\frac{n-1}{2}(\bmod n)\right\}, \\
R_{5}= & \mathscr{R}\left\{t_{k} u_{k}\right\}=V\left(H_{4}^{6}\right)-\left\{s_{r} \mid r \equiv k, k+1(\bmod 4)\right\} \cup\left\{t_{r} \mid r \equiv k+1, k+2(\bmod 4)\right\} \cup
\end{aligned}
$$




$$
\begin{aligned}
& \cdot\left\{v_{r} \mid r \equiv k+2(\bmod 4)\right\} \cup\left\{u_{r} \mid r \equiv k+2, k+3(\bmod 4)\right\} \\
R_{6}= & \mathscr{R}\left\{s_{k} v_{k}\right\}=V\left(H_{n}^{6}\right)-\left\{s_{r} \mid r \equiv k+1, k+2, \ldots, k+\frac{n-1}{2}(\bmod n)\right\} \cup \\
& \cdot\left\{t_{r} \mid r \equiv k+2, k+3, \ldots, k+\frac{n+1}{2}(\bmod n)\right\} \cup\left\{v_{r} \mid r \equiv k+1+\frac{n-1}{2}, k+2+\frac{n-1}{2}, \ldots, k+n-1(\bmod n)\right\} \\
& \cup\left\{u_{r} \mid r \equiv k(\bmod n), r \equiv k+1+\frac{n-1}{2}, k+2+\frac{n-1}{2}, \ldots, k+n-1(\bmod n)\right\} .
\end{aligned}
$$

The cardinality of each of the local resolving neighborhoods $\overline{R_{1}}, R_{1}, R_{2}, R_{3}, R_{4}, R_{5}$, and $R_{6}$ for each $1 \leq k \leq n$ is $2 n, 4 n-3,4 n-1,4 n-3,4 n-1,2 n+1$, and $2 n+1$, respectively. Since cardinality of $\overline{R_{1}}$ is $2 n$ which is less than the cardinality of all other resolving neighborhoods and $\cup_{i=1}^{6} R_{i}=V\left(H_{n}^{6}\right)$, this implies that $\left|\overline{R_{j}} \cap \cup_{i=1}^{6} R_{i}\right| \geq\left|R_{i}\right|$. Now, we construct a mapping $\vartheta: V\left(H_{n}^{6}\right) \longrightarrow[0,1]$ as

$$
\vartheta\left(u_{k}\right)=\vartheta\left(s_{k}\right)=\frac{1}{2 n} \text {. }
$$

Since $2 n$ is a minimal cardinal number, there must exist a minimal resolving function $\vartheta^{\prime}$ such that $\left|\vartheta^{\prime}\right| \leq|\vartheta|$. Hence,

$$
\mathscr{D i m}_{l f}\left(H_{n}^{6}\right) \leq \sum_{i=1}^{4 n} \frac{1}{2 n}=2
$$

Lemma 4. Let $H_{n}^{7}$ be a hexagonal planar circular network for $n \geq 5$ with $n \equiv 1(\bmod 2)$.

(1) Then, for $1 \leq k \leq n,\left|\mathscr{R}_{k}\right|=\left|\mathscr{R}\left\{s_{k} t_{k}\right\}\right|=\left|\mathscr{R}\left\{s_{k} t_{k+1}\right\}\right|=$ $\left|\mathscr{R}\left\{t_{k} u_{k}\right\}\right|=\left|\mathscr{R}\left\{t_{k} u_{k+1}\right\}\right|=2 n+3$ with $t_{n+1}=t_{1}$, $u_{n+1}=u_{1}$ and $\left|\cup_{k=1}^{n} \mathscr{R}_{k}\right|=4 n$.

(2) $\left|\mathscr{R}_{k}\right|<\left|\mathscr{R}\left\{u_{k} v_{k}\right\}\right|$ and $\left|\mathscr{R}\left\{u_{k} v_{k}\right\} \cap \cup_{k=1}^{n} \mathscr{R}_{k}\right| \geq\left|\mathscr{R}_{k}\right|$.

(3) $\left|\mathscr{R}_{k}\right|<\left|\mathscr{R}\left\{u_{k} v_{k-1}\right\}\right|$ and $\left|\mathscr{R}\left\{u_{k} v_{k-1}\right\} \cap \cup_{k=1}^{n} \mathscr{R}_{k}\right|$ $\geq\left|\mathscr{R}_{k}\right|$ with $v_{n}=v_{0}$.

(4) $\left|\mathscr{R}_{k}\right|<\left|\mathscr{R}\left\{t_{k} t_{k+1}\right\}\right|$ and $\left|\mathscr{R}\left\{t_{k} t_{k+1}\right\} \cap \cup_{k=1}^{n} \mathscr{R}_{k}\right| \geq\left|\mathscr{R}_{k}\right|$, $t_{n+1}=t_{1}$.

Proof

(1) The set of local resolving neighborhoods of $s_{k} t_{k}$, $s_{k} t_{k+1}, t_{k} u_{k}$ and $t_{k} u_{k+1}$ with $u_{n+1}=u_{1}, t_{n+1}=t_{1}$ is $\mathscr{R}\left\{s_{k} t_{k}\right\}=V\left(H_{n}^{7}\right)-\left\{s_{r} \mid r \equiv k+1, k+2, \ldots, k+(n+1 /\right.$ 2) $-1(\bmod n)\}\left\{\bigcup t_{r} \mid r \equiv k+1, k+2, \ldots, k+(n+1 / 2)-\right.$ $1(\bmod n)\}\left\{\bigcup u_{r} \mid r \equiv k+2, k+3, \ldots, k+(n+3 / 2)-1\right.$ $(\bmod n)\}\left\{\bigcup v_{r} \mid r \equiv k+2, k+3, \ldots, k+(n+1 / 2)-1\right.$ $(\bmod n)\}, \mathscr{R}\left\{s_{k} t_{k+1}\right\}=V\left(H_{n}^{7}\right)-\left\{s_{r} \mid r \equiv k+1+(n-1 /\right.$ $2), k+2+\quad(n-1 / 2), \ldots, k+n-1(\bmod n)\} \bigcup\left\{t_{r} \mid r \equiv k\right.$ $(\bmod n), r \equiv k+2+(n-1 / 2), k+3+(n-1 / 2), \ldots, k+$ $n-1(\bmod n)\} \bigcup\left\{u_{r} \mid r \equiv k(\bmod n), r \equiv k+2+(n-1 /\right.$ $2), k+3+(n-1 / 2), \ldots, k+n-1(\bmod n)\} \bigcup\left\{v_{r} \mid r \equiv k+\right.$ $2+(n-1 / 2), k+3+(n-1 / 2), \ldots, k+n-1(\bmod n)\}$, $\mathscr{R}\left\{t_{k} u_{k}\right\}=V\left(H_{n}^{7}\right)-\left\{s_{r} \mid r \equiv k+(n-1 / 2), k+1+(n-1\right.$ $12), \ldots, k+n-2(\bmod n)\} \bigcup\left\{t_{r} \mid r \equiv k+(n-1 / 2)+1, k\right.$ $+2+(n-1 / 2), \ldots, k+n-1(\bmod n)\} \bigcup\left\{u_{r} \mid r \equiv k+1+\right.$ $(n-1 / 2), k+2+(n-1 / 2), \ldots, k+n-1 \quad(\bmod n)\} \bigcup$ $\left\{v_{r} \mid r \equiv k+1+(n-1 / 2), k+2+(n-1 / 2), \ldots, k+n-2\right.$ $(\bmod n)\}$, and $\mathscr{R}\left\{t_{k} u_{k+1}\right\}=V\left(H_{n}^{7}\right)-\left\{s_{r} \mid r \equiv k+1, k+\right.$ $2, \ldots, k+(n+1 / 2)-1(\bmod n)\} \bigcup\left\{t_{r} \mid r \equiv k+1, \quad k+2\right.$, $\ldots, k+(n+1 / 2)-1(\bmod n)\} \bigcup\left\{u_{r} \mid r \equiv k+2, k+3, \ldots\right.$, $k+(n+3 / 2)-1(\bmod n)\} \bigcup\left\{v_{r} \mid r \equiv k+2, k+3, \ldots, k+\right.$ $(n+1 / 2)-1(\bmod n)\}$. Clearly, $\cup_{k=1}^{n} \mathscr{R}_{k}=V\left(H_{n}^{7}\right)$. Therefore, $\left|\cup_{k=1}^{n} \mathscr{R}_{k}\right|=4 n$.

(2) The set of local resolving neighborhoods of $u_{k} v_{k}$ is $\mathscr{R}\left\{u_{k} v_{k}\right\}=V\left(H_{n}^{7}\right)-\left\{s_{r} \mid r \equiv k+1, k+2, \ldots, k+(n-\right.$ $1 / 2)-1(\bmod n)\} \bigcup\left\{t_{r} \mid r \equiv k+1, k+2, \ldots, k+(n-\right.$ $1 / 2)-1(\bmod n)\} \bigcup\left\{u_{r} \mid r \equiv k+2, k+3, \ldots, k+(n+\right.$ $1 / 2)-1 \quad(\bmod n)\} \bigcup\left\{v_{r} \mid r \equiv k+2, k+3, \ldots, \quad k+(n\right.$ $+1 / 2)-1(\bmod n)\}$. Hence, $\left|\mathscr{R}\left\{u_{k} v_{k}\right\}\right|=2 n+5$. Since $\left|\mathscr{R}_{k}\right|=2 n+3<\left|\mathscr{R}\left\{u_{k} v_{k}\right\}\right|=2 n+5, \quad \mid \mathscr{R}\left\{u_{k} v_{k}\right\} \cap$ $\cup_{k=1}^{n} \mathscr{R}_{k}|=2 n+3 \geq| \mathscr{R}_{k} \mid$.

(3) The set of local resolving neighborhoods of $u_{k} v_{k-1}$ is $\mathscr{R}\left\{u_{k} v_{k-1}\right\}=V\left(H_{n}^{7}\right)-\left\{s_{r} \mid r \equiv k+(n-1 / 2), k+1+\right.$ $(n-1 / 2), k+2+(n-1 / 2), \ldots, k+n-3 \quad(\bmod n)\}$ $\bigcup\left\{t_{r} \mid r \equiv k+(n-1 / 2), k+1+(n-1 / 2), \ldots, k+n-\right.$ $2(\bmod n)\} \bigcup\left\{u_{r} \mid r \equiv k+1+(n-1 / 2), k+2+(n-1\right.$ $12), \ldots, k+n-2 \quad(\bmod n)\} \bigcup\left\{v_{r} \mid r \equiv k+(n-1 / 2)\right.$, $k+1+(n-1 / 2), \ldots, k+n-3(\bmod n)\}$. Hence, $\left|\mathscr{R}\left\{u_{k} v_{k-1}\right\}\right|=2 n+5$. Since $\left|\mathscr{R}_{k}\right|=2 n+3<\mid \mathscr{R}$ $\left\{u_{k} v_{k-1}\right\}|=2 n+5,| \mathscr{R}\left\{u_{k} v_{k-1}\right\} \cap \cup_{k=1}^{n} \mathscr{R}_{k} \mid=2 n+3 \geq$ $\left|\mathscr{R}_{k}\right|$.

(4) The set of local resolving neighborhoods of $t_{k} t_{k+1}$, with $v_{n+1}=v_{1}$, is $\mathscr{R}\left\{t_{k} t_{k+1}\right\}=V\left(H_{n}^{7}\right)-\left\{s_{r} \mid r \equiv k(\bmod \right.$ $n)\} \bigcup\left\{t_{r} \mid r \equiv k+(n+3 / 2)-1(\bmod n)\right\} \bigcup\left\{u_{r} \mid r \equiv k+1\right.$ $(\bmod n)\} \bigcup\left\{v_{r} \mid r \equiv k(\bmod n), r \equiv k+1(\bmod n), r \equiv\right.$ $k+(n+3 / 2)-1(\bmod n)\}$. Hence, $\left|\mathscr{R}\left\{t_{k} t_{k+1}\right\}\right|=4 n-$ 6. Since $\left|\mathscr{R}_{k}\right|=2 n+3<\left|\mathscr{R}\left\{t_{k} t_{k+1}\right\}\right|=4 n-6, \quad \mid \mathscr{R}\left\{u_{k}\right.$ $\left.v_{k-1}\right\} \cap \cup_{k=1}^{n} \mathscr{R}_{k}|=2 n+3 \geq| \mathscr{R}_{k} \mid$.

Theorem 5. If $H_{n}^{7}$ is a hexagonal planar circular network, then

$$
\mathscr{D i m}_{l f}\left(H_{n}^{7}\right) \leq \begin{cases}2, & n=3 \\ \frac{4 n}{2 n+3}, & n \geq 5\end{cases}
$$

Proof. The local resolving neighborhoods are

Case (a): for $n=3,1 \leq k \leq 3$, by Lemma 4 , 


$$
\begin{aligned}
\overline{R_{1}}= & \mathscr{R}\left\{t_{k} t_{k+1}\right\}=V\left(H_{3}^{6}\right)-\left\{s_{r} \mid r \equiv k(\bmod 3)\right\} \cup\left\{t_{r} \mid r \equiv k+\frac{n+3}{2}-1(\bmod 3)\right\} \cup \\
& \cdot\left\{u_{r} \mid r \equiv k+1(\bmod 3)\right\} \cup\left\{v_{r} \mid r \equiv k, k+1, k+\frac{n+3}{2}-1(\bmod 3)\right\}, \\
R_{1}= & \mathscr{R}\left\{s_{k} t_{k}\right\}=V\left(H_{3}^{6}\right)-\left\{s_{r} \mid r \equiv k+1(\bmod 3)\right\} \cup\left\{t_{r} \mid r \equiv k+1(\bmod 3)\right\} \cup\left\{u_{r} \mid r \equiv k+2(\bmod 3)\right\}, \\
R_{2}= & \mathscr{R}\left\{s_{k} t_{k+1}\right\}=V\left(H_{3}^{6}\right)-\left\{s_{r} \mid r \equiv k+2(\bmod 3)\right\} \cup\left\{t_{r} \mid r \equiv k(\bmod 3)\right\} \cup\left\{u_{r} \mid r \equiv k(\bmod 3)\right\}, \\
R_{3}= & \mathscr{R}\left\{t_{k} u_{k}\right\}=V\left(H_{3}^{6}\right)-\left\{s_{r} \mid r \equiv k+1(\bmod 3)\right\} \cup\left\{t_{r} \mid r \equiv k+2(\bmod 3)\right\} \cup\left\{u_{r} \mid r \equiv k+2(\bmod 3)\right\}, \\
R_{4}= & \mathscr{R}\left\{t_{k} u_{k+1}\right\}=V\left(H_{3}^{6}\right)-\left\{s_{r} \mid r \equiv k+1(\bmod 3)\right\} \cup\left\{t_{r} \mid r \equiv k+1(\bmod 3)\right\} \cup\left\{u_{r} \mid r \equiv k+2(\bmod 3)\right\}, \\
R_{5}= & \mathscr{R}\left\{u_{k} v_{k}\right\}=V\left(H_{3}^{6}\right)-\left\{s_{r} \mid r \equiv k+1(\bmod 3)\right\} \cup\left\{t_{r} \mid r \equiv k+1, k+2(\bmod 3)\right\} \cup \\
& \cdot\left\{u_{r} \mid r \equiv k+2(\bmod 3)\right\} \cup\left\{v_{r} \mid r \equiv k+2(\bmod 3)\right\}, \\
R_{6}= & \mathscr{R}\left\{u_{k} v_{k-1}\right\}=V\left(H_{3}^{6}\right)-\left\{t_{r} \mid r \equiv k+1(\bmod 3)\right\} .
\end{aligned}
$$

The cardinality of each of the local resolving neighborhoods $\overline{R_{1}}, R_{1}, R_{2}, R_{3}, R_{4}, R_{5}$, and $R_{6}$ for each $k=1,2,3$ is $6,9,9,9,9,11$, and 11 , respectively. Since cardinality of $\overline{R_{1}}$ is 6 which is less than the cardinality of all other resolving neighborhoods and $\cup_{i=1}^{6} R_{i}=$ $V\left(H_{3}^{6}\right)$, this implies that $\left|\overline{R_{j}} \cap \cup_{i=1}^{6} R_{i}\right| \geq\left|R_{i}\right|$. Now, we construct a mapping $\vartheta: V\left(H_{3}^{6}\right) \longrightarrow[0,1]$ as

$$
\vartheta\left(t_{k}\right)=\vartheta\left(t_{k+1}\right)=\frac{1}{6} .
$$

Since 6 is a minimal cardinal number, there must exist a minimal resolving function $\vartheta^{\prime}$ such that $\left|\vartheta^{\prime}\right| \leq|\vartheta|$. Hence,

$$
\mathscr{D i m}_{l f}\left(H_{3}^{6}\right) \leq \sum_{i=1}^{12} \frac{1}{6}=2 \text {. }
$$

Case (b): for $n \geq 5,1 \leq k \leq 5$, by Lemma 8 , we have

$$
\begin{aligned}
\overline{R_{1}}= & \mathscr{R}\left\{s_{k} t_{k}\right\}=V\left(H_{n}^{7}\right)-\left\{s_{r} \mid r \equiv k+1, k+2, \ldots, k+\frac{n+1}{2}-1(\bmod n)\right\} \cup \\
& \cdot\left\{t_{r} \mid r \equiv k+1, k+2, \ldots, k+\frac{n+1}{2}-1(\bmod n)\right\} \cup\left\{u_{r} \mid r \equiv k+2, k+3, \ldots, k+\frac{n+3}{2}-1(\bmod n)\right\} \\
& \cup\left\{v_{r} \mid r \equiv k+2, k+3, \ldots, k+\frac{n+1}{2}-1(\bmod n)\right\}, \\
\overline{R_{2}}= & \mathscr{R}\left\{s_{k} t_{k+1}\right\}=V\left(H_{n}^{7}\right)-\left\{s_{r} \mid r \equiv k+1+\frac{n-1}{2}, k+2+\frac{n-1}{2}, \ldots, k+n-1(\bmod n)\right\} \cup \\
& \cdot\left\{t_{r} \mid r \equiv k(\bmod n), r \equiv k+2+\frac{n-1}{2} k+3+\frac{n-1}{2}, \ldots, k+n-1(\bmod n)\right\} \cup \\
& \cdot\left\{u_{r} \mid r \equiv k(\bmod n), r \equiv k+2+\frac{n-1}{2}, k+3+\frac{n-1}{2}, \ldots, k+n-1(\bmod n)\right\} \cup \\
& \cdot\left\{v_{r} \mid r \equiv k+2+\frac{n-1}{2}, k+3+\frac{n-1}{2}, \ldots, k+n-1(\bmod n)\right\}, \\
\overline{R_{3}}= & \mathscr{R}\left\{t_{k} u_{k}\right\}=V\left(H_{n}^{7}\right)-\left\{s_{r} \mid r \equiv k+\frac{n-1}{2}, k+1+\frac{n-1}{2}, \ldots, k+n-2(\bmod n)\right\} \cup
\end{aligned}
$$




$$
\begin{aligned}
& \cdot\left\{t_{r} \mid r \equiv k+\frac{n-1}{2}+1, k+2+\frac{n-1}{2}, \ldots, k+n-1(\bmod n)\right\} \cup \\
& \cdot\left\{u_{r} \mid r \equiv k+1+\frac{n-1}{2}, k+2+\frac{n-1}{2}, \ldots, k+n-1(\bmod n)\right\} \cup \\
& \cdot\left\{v_{r} \mid r \equiv k+1+\frac{n-1}{2}, k+2+\frac{n-1}{2}, \ldots, k+n-2(\bmod n)\right\}, \\
& \overline{R_{4}}=\mathscr{R}\left\{t_{k} u_{k+1}\right\}=V\left(H_{n}^{7}\right)-\left\{s_{r} \mid r \equiv k+1, k+2, \ldots, k+\frac{n+1}{2}-1(\bmod n)\right\} \cup \\
& \cdot\left\{t_{r} \mid r \equiv k+1, k+2, \ldots, k+\frac{n+1}{2}-1(\bmod n)\right\} \cup\left\{u_{r} \mid r \equiv k+2, k+3, \ldots, k+\frac{n+3}{2}-1(\bmod n)\right\} \\
& \cup\left\{v_{r} \mid r \equiv k+2, k+3, \ldots, k+\frac{n+1}{2}-1(\bmod n)\right\}, \\
& R_{1}=\mathscr{R}\left\{u_{k} v_{k}\right\}=V\left(H_{n}^{7}\right)-\left\{s_{r} \mid r \equiv k+1, k+2, \ldots, k+\frac{n-1}{2}-1(\bmod n)\right\} U \\
& \cdot\left\{t_{r} \mid r \equiv k+1, k+2, \ldots, k+\frac{n-1}{2}-1(\bmod n)\right\} \cup\left\{u_{r} \mid r \equiv k+2, k+3, \ldots, k+\frac{n+1}{2}-1(\bmod n)\right\} \\
& \cup\left\{v_{r} \mid r \equiv k+2, k+3, \ldots, k+\frac{n+1}{2}-1(\bmod n)\right\} \text {, } \\
& R_{2}=\mathscr{R}\left\{u_{k} v_{k-1}\right\}=V\left(H_{n}^{7}\right)-\left\{s_{r} \mid r \equiv k+\frac{n-1}{2}, k+1+\frac{n-1}{2}, k+2+\frac{n-1}{2}, \ldots, k+n-3(\bmod n)\right\} \\
& \cup\left\{t_{r} \mid r \equiv k+\frac{n-1}{2}, k+1+\frac{n-1}{2}, \ldots, k+n-2(\bmod n)\right\} \cup \\
& \cdot\left\{u_{r} \mid r \equiv k+1+\frac{n-1}{2}, k+2+\frac{n-1}{2}, \ldots, k+n-2(\bmod n)\right\} \\
& \cup\left\{v_{r} \mid r \equiv k+\frac{n-1}{2}, k+1+\frac{n-1}{2}, \ldots, k+n-3(\bmod n)\right\} \\
& R_{3}=\mathscr{R}\left\{t_{k} t_{k+1}\right\}=V\left(H_{n}^{7}\right)-\left\{s_{r} \mid r \equiv k(\bmod n)\right\} \cup\left\{t_{r} \mid r \equiv k+\frac{n+3}{2}-1(\bmod n)\right\} \\
& \cup\left\{u_{r} \mid r \equiv k+1(\bmod n)\right\} \cup\left\{v_{r} \mid r \equiv k(\bmod n), r \equiv k+1(\bmod n), r \equiv k+\frac{n+3}{2}-1(\bmod n)\right\} .
\end{aligned}
$$

The cardinality of each of the local resolving neighborhoods $\overline{R_{1}}, \overline{R_{2}}, \overline{R_{3}}, \overline{R_{4}}, R_{1}, R_{2}$, and $R_{3}$ for each $1 \leq k \leq n$ is $2 n+3,2 n+3,2 n+3,2 n+3,2 n+5$, and $4 n-6$, respectively. Since cardinality of $\overline{R_{i}}, 1 \leq i \leq 4$ is $2 n+3$ which is less than the cardinality of all other resolving neighborhoods and $\cup_{i=1}^{3} R_{i}=V\left(H_{n}^{7}\right)$, this implies that $\left|\overline{R_{j}} \cap \cup_{i=1}^{3} R_{i}\right| \geq\left|R_{i}\right|$. Now, we construct a mapping ९: $V\left(H_{n}^{7}\right) \longrightarrow[0,1]$ as

$$
\vartheta\left(t_{k}\right)=\vartheta\left(s_{k}\right)=\vartheta\left(t_{k+1}\right)=\vartheta\left(u_{k}\right)=\vartheta\left(u_{k+1}\right)=\frac{1}{2 n+3} .
$$

Since $2 n+3$ is a minimal cardinal number, there must exist a minimal resolving function $\vartheta^{\prime}$ such that $\left|\vartheta^{\prime}\right| \leq|\vartheta|$. Hence,

$$
\mathscr{D i m}_{l f}\left(H_{n}^{7}\right) \leq \sum_{i=1}^{4 n} \frac{1}{2 n+3}=\frac{4 n}{2 n+3}
$$

Lemma 5. Let $H_{n}^{8}$ be a hexagonal planar circular network for $n \geq 5$ with $n \equiv 1(\bmod 2)$.

(1) Then, for $1 \leq k \leq n, \quad\left|\mathscr{R}_{k}\right|=\left|\mathscr{R}\left\{u_{k} v_{k}\right\}\right|=8$ and $\left|\cup_{k=1}^{n} \mathscr{R}_{k}\right|=4 n$ with $u_{n+1}=u_{1}$.

(2) $\left|\mathscr{R}_{k}\right|<\left|\mathscr{R}\left\{s_{k} t_{k}\right\}\right|$ and $\left|\mathscr{R}\left\{s_{k} t_{k}\right\} \cap \cup_{k=1}^{n} \mathscr{R}_{k}\right| \geq\left|\mathscr{R}_{k}\right|$.

(3) $\left|\mathscr{R}_{k}\right|<\left|\mathscr{R}\left\{t_{k} u_{k}\right\}\right|$ and $\left|\mathscr{R}\left\{t_{k} u_{k}\right\} \cap \cup_{k=1}^{n} \mathscr{R}_{k}\right| \geq\left|\mathscr{R}_{k}\right|$.

(4) $\left|\mathscr{R}_{k}\right|<\left|\mathscr{R}\left\{t_{k} t_{k+1}\right\}\right|$ and $\left|\mathscr{R}\left\{t_{k} t_{k+1}\right\} \cap \cup_{k=1}^{n} \mathscr{R}_{k}\right| \geq\left|\mathscr{R}_{k}\right|$, with $t_{n+1}=t_{1}$. 
(5) $\left|\mathscr{R}_{k}\right|<\left|\mathscr{R}\left\{t_{k} v_{k}\right\}\right|$ and $\left|\mathscr{R}\left\{t_{k} v_{k}\right\} \cap \cup_{k=1}^{n} \mathscr{R}_{k}\right| \geq\left|\mathscr{R}_{k}\right|$.

(6) $\left|\mathscr{R}_{k}\right|<\left|\mathscr{R}\left\{u_{k} v_{k-1}\right\}\right| \quad$ and $\quad\left|\mathscr{R}\left\{u_{k} v_{k-1}\right\} \cap \cup_{k=1}^{n} \mathscr{R}_{k}\right|$ $\geq\left|\mathscr{R}_{k}\right|$, with $v_{n}=v_{0}$.

(7) $\left|\mathscr{R}_{k}\right|<\left|\mathscr{R}\left\{s_{k} t_{k+1}\right\}\right|$ and $\left|\mathscr{R}\left\{s_{k} t_{k+1}\right\} \cap \cup_{k=1}^{n} \mathscr{R}_{k}\right| \geq\left|\mathscr{R}_{k}\right|$, with $t_{k+1}=t_{1}$.

Theorem 6. If $H_{n}^{8}$ is a hexagonal planar circular network, then

$$
\mathscr{D i m m}_{l f}\left(H_{n}^{8}\right) \leq \begin{cases}2, & n=3, \\ \frac{n}{2}, & n \geq 5 .\end{cases}
$$

Lemma 6. Let $H_{n}^{9}$ be a hexagonal planar circular network for $n \geq 3$ with $n \equiv 1(\bmod 2)$.

(1) Then, for $1 \leq k \leq n, \quad\left|\mathscr{R}_{k}\right|=\left|\mathscr{R}\left\{t_{k} v_{k}\right\}\right|=4$, and $\left|\cup_{k=1}^{n} \mathscr{R}_{k}\right|=4 n$.

(2) $\left|\mathscr{R}_{k}\right|<\left|\mathscr{R}\left\{t_{k} u_{k}\right\}\right|$ and $\left|\mathscr{R}\left\{t_{k} u_{k}\right\} \cap \cup_{k=1}^{n} \mathscr{R}_{k}\right| \geq\left|\mathscr{R}_{k}\right|$.

(3) $\left|\mathscr{R}_{k}\right|<\left|\mathscr{R}\left\{u_{k} v_{k-1}\right\}\right|$ and $\left|\mathscr{R}\left\{u_{k} v_{k-1}\right\} \cap \cup_{k=1}^{n} \mathscr{R}_{k}\right| \geq$ $\left|\mathscr{R}_{k}\right|$ with $v_{n}=u_{0}$.

(4) $\left|\mathscr{R}_{k}\right|<\left|\mathscr{R}\left\{u_{k} v_{k}\right\}\right|$ and $\left|\mathscr{R}\left\{u_{k} v_{k}\right\} \cap \cup_{k=1}^{n} \mathscr{R}_{k}\right| \geq\left|\mathscr{R}_{k}\right|$.

(5) $\left|\mathscr{R}_{k}\right|<\left|\mathscr{R}\left\{s_{k} t_{k+1}\right\}\right|$ and $\left|\mathscr{R}\left\{s_{k} t_{k+1}\right\} \cap \cup \cup_{k=1}^{n} \mathscr{R}_{k}\right| \geq\left|\mathscr{R}_{k}\right|$, with $t_{n+1}=t_{1}$.

(6) $\left|\mathscr{R}_{k}\right|<\left|\mathscr{R}\left\{s_{k} t_{k}\right\}\right|$ and $\left|\mathscr{R}\left\{s_{k} t_{k}\right\} \cap \cup_{k=1}^{n} \mathscr{R}_{k}\right| \geq\left|\mathscr{R}_{k}\right|$.

(7) $\left|\mathscr{R}_{k}\right|<\left|\mathscr{R}\left\{t_{k} u_{k+1}\right\}\right| \quad$ and $\left|\mathscr{R}\left\{t_{k} u_{k+1}\right\} \cap \cup_{k=1}^{n} \mathscr{R}_{k}\right| \geq\left|\mathscr{R}_{k}\right|$, with $u_{n+1}=u_{1}$.

Proof

(1) The set of local resolving neighborhoods of $t_{k} v_{k}$ is $\mathscr{R}\left\{t_{k}\right.$ $\left.v_{k}\right\}=V\left(H_{n}^{9}\right)-\left\{s_{r} \mid r \equiv k+1, k+2, \ldots, k+n-(\bmod n)\right\}$ $\bigcup\left\{t_{r} \mid r \equiv k+1, k+2, \ldots, k+n-2(\bmod n)\right\} \bigcup\left\{u_{r} \mid\right.$ $r \equiv k, k+1, k+2, \ldots, k+n \quad-1(\bmod n)\} \quad \bigcup\left\{v_{r} \mid r \equiv k\right.$
$+1, k+2, \ldots, k+n-1(\bmod n)\} . \quad$ Clearly, $\quad \cup_{k=1}^{n} \mathscr{R}_{k}=$ $V\left(H_{n}^{9}\right)$. Therefore, $\left|\cup_{k=1}^{n} \mathscr{R}_{k}\right|=4 n$.

(2) The set of local resolving neighborhoods of $s_{k} t_{k+1}$ is $\mathscr{R}$ $\left\{s_{k} t_{k+1}\right\}=V\left(H_{n}^{9}\right)-\left\{v_{r} \mid r \equiv k+1(\bmod n)\right\}$. Hence, $\left|\mathscr{R}\left\{s_{k} t_{k+1}\right\}\right|=4 n-1$. Since $\left|\mathscr{R}_{k}\right|=4<\left|\mathscr{R}\left\{s_{k} t_{k+1}\right\}\right|$ $=4 n-1,\left|\mathscr{R}\left\{s_{k} t_{k+1}\right\} \cap \cup_{k=1}^{n} \mathscr{R}_{k}\right|=4 n-1 \geq\left|\mathscr{R}_{k}\right|$.

(3) The set of local resolving neighborhoods of $s_{k} t_{k}$ is $\mathscr{R}\left\{s_{k} t_{k}\right\}=V\left(H_{n}^{9}\right)-\left\{v_{r} \mid r \equiv k(\bmod n)\right\}$. Hence, $\mid \mathscr{R}$ $\left\{s_{k} t_{k}\right\} \mid=4 n-1$. Since $\left|\mathscr{R}_{k}\right|=4<\left|\mathscr{R}\left\{s_{k} t_{k}\right\}\right|=4 n-1$, $\left|\mathscr{R}\left\{s_{k} t_{k}\right\} \cap \cup_{k=1}^{n} \mathscr{R}_{k}\right|=4 n-1 \geq\left|\mathscr{R}_{k}\right|$.

(4) The set of local resolving neighborhoods of $t_{k} u_{k}$ is $\mathscr{R}\left\{t_{k} u_{k}\right\}=V\left(H_{n}^{9}\right)-\left\{s_{r} \mid r \equiv k(\bmod n), r \equiv k+n-1\right.$ $(\bmod n)\} \cup\left\{t_{r} \mid r \equiv k(\bmod n)\right\}$. Hence, $\left|\mathscr{R}\left\{t_{k} u_{k}\right\}\right|$ $=4 n-1$. Since $\left|\mathscr{R}_{k}\right|=4<\left|\mathscr{R}\left\{t_{k} u_{k}\right\}\right|=4 n-1, \mid \mathscr{R}\left\{t_{k}\right.$ $\left.u_{k}\right\} \cap \cup_{k=1}^{n} \mathscr{R}_{k}|=4 n-1 \geq| \mathscr{R}_{k} \mid$.

(5) The set of local resolving neighborhoods of $t_{k} u_{k+1}$ is $\mathscr{R}\left\{t_{k} u_{k+1}\right\}=V\left(H_{n}^{9}\right)-\left\{v_{r} \mid r \equiv k(\bmod n)\right\}$. Hence, $\left|\mathscr{R}\left\{t_{k} u_{k+1}\right\}\right|=4 n-1$. Since $\left|\mathscr{R}_{k}\right|=4<\left|\mathscr{R}\left\{t_{k} u_{k+1}\right\}\right|$ $=4 n-1,\left|\mathscr{R}\left\{t_{k} u_{k+1}\right\} \cap \cup_{k=1}^{n} \mathscr{R}_{k}\right|=4 n-1 \geq\left|\mathscr{R}_{k}\right|$.

(6) The set of local resolving neighborhoods of $u_{k} v_{k-1}$ is $\mathscr{R}\left\{u_{k} v_{k-1}\right\}=V\left(H_{n}^{9}\right)-\left\{v_{r} \mid r \equiv k+n-2(\bmod n), r \equiv\right.$ $k+n-1(\bmod n)\} \quad \cup\left\{t_{r} \mid r \equiv k+n-1(\bmod n)\right\}$. Hence, $\quad\left|\mathscr{R}\left\{u_{k} v_{k-1}\right\}\right|=4 n-3$. Since $\left|\mathscr{R}_{k}\right|=4<$ $\left|\mathscr{R}\left\{u_{k} v_{k-1}\right\}\right|=4 n-3, \quad\left|\mathscr{R}\left\{u_{k} v_{k-1}\right\} \cap \cup_{k=1}^{n} \mathscr{R}_{k}\right|=4 n$ $-3 \geq\left|\mathscr{R}_{k}\right|$.

(7) The set of local resolving neighborhoods of $u_{k} v_{k}$ is $\mathscr{R}\left\{u_{k} v_{k}\right\}=V\left(H_{n}^{9}\right)-\left\{v_{r} \mid r \equiv k(\bmod n), r \equiv k+n-1\right.$ $(\bmod n)\} \cup\left\{t_{r} \mid r \equiv k+n-1 \quad(\bmod n)\right\}$. Hence, $\mid \mathscr{R}$ $\left\{u_{k} v_{k}\right\} \mid=4 n-3$. Since $\left|\mathscr{R}_{k}\right|=4<\left|\mathscr{R}\left\{u_{k} v_{k}\right\}\right|=$ $4 n-3,\left|\mathscr{R}\left\{u_{k} v_{k}\right\} \cap \cup_{k=1}^{n} \mathscr{R}_{k}\right|=4 n-3 \geq\left|\mathscr{R}_{k}\right|$.

Theorem 7. If $H_{n}^{9}$ is a hexagonal planar circular network for $n \geq 3$ with $n \equiv 1(\bmod 2)$, then $\mathscr{D i m}_{l f}\left(H_{n}^{9}\right) \leq n$.

Proof. When $n \geq 3$ with $n \equiv 1(\bmod 2)$, by Lemma 6 , the local resolving neighborhoods are

$$
\begin{aligned}
\overline{R_{1}}= & \mathscr{R}\left\{t_{k} v_{k}\right\}=V\left(H_{n}^{9}\right)-\left\{s_{r} \mid r \equiv k+1, k+2, \ldots, k+n-2(\bmod n)\right\} \cup \\
& \cdot\left\{t_{r} \mid r \equiv k+1, k+2, \ldots, k+n-2(\bmod n)\right\} \cup\left\{u_{r} \mid r \equiv k, k+1, k+2, \ldots, k+n-1(\bmod n)\right\} \cup \\
& \cdot\left\{v_{r} \mid r \equiv k+1, k+2, \ldots, k+n-1(\bmod n)\right\}, \\
R_{1}= & \mathscr{R}\left\{s_{k} t_{k+1}\right\}=V\left(H_{n}^{9}\right)-\left\{v_{r} \mid r \equiv k+1(\bmod n)\right\}, \\
R_{2}= & \mathscr{R}\left\{s_{k} t_{k}\right\}=V\left(H_{n}^{9}\right)-\left\{v_{r} \mid r \equiv k(\bmod n)\right\}, \\
R_{3}= & \mathscr{R}\left\{t_{k} u_{k}\right\}=V\left(H_{n}^{9}\right)-\left\{s_{r} \mid r \equiv k(\bmod n), r \equiv k+n-1(\bmod n)\right\} \cup\left\{t_{r} \mid r \equiv k(\bmod n)\right\}, \\
R_{4}= & \mathscr{R}\left\{t_{k} u_{k+1}\right\}=V\left(H_{n}^{9}\right)-\left\{v_{r} \mid r \equiv k(\bmod n)\right\}, \\
R_{5}= & \mathscr{R}\left\{u_{k} v_{k-1}\right\}=V\left(H_{n}^{9}\right)-\left\{v_{r} \mid r \equiv k+n-2(\bmod n), r \equiv k+n-1(\bmod n)\right\} \cup\left\{t_{r} \mid r \equiv k+n-1(\bmod n)\right\}, \\
R_{6}= & \mathscr{R}\left\{u_{k} v_{k}\right\}=V\left(H_{n}^{9}\right)-\left\{v_{r} \mid r \equiv k(\bmod n), r \equiv k+n-1(\bmod n)\right\} \cup\left\{t_{r} \mid r \equiv k+n-1(\bmod n)\right\} .
\end{aligned}
$$

The cardinality of each of the local resolving neighborhoods $\overline{R_{1}}, \overline{R_{2}}, R_{1}, R_{2}, R_{3}, R_{4}, R_{5}$, and $R_{6}$ for each $1 \leq k \leq n$ is $4,4 n-1,4 n-1,4 n-1,4 n-1,4 n-1,4 n-3$, and $4 n-3$, respectively. Since cardinality of $\overline{R_{1}}$ is 4 which is less than the 
cardinality of all other resolving neighborhoods and $\cup_{i=1}^{6} R_{i}=V\left(H_{n}^{9}\right)$, this implies that $\left|\overline{R_{j}} \cap \cup_{i=1}^{6} R_{i}\right| \geq\left|R_{i}\right|$. Now, we construct a mapping $\vartheta: V\left(H_{n}^{9}\right) \longrightarrow[0,1]$ as

$$
\vartheta\left(t_{k}\right)=\vartheta\left(v_{k}\right)=\frac{1}{4} .
$$

Since 4 is a minimal cardinal number, there must exist a minimal resolving function $\vartheta^{\prime}$ such that $\left|\vartheta^{\prime}\right| \leq|\vartheta|$. Hence,

$$
\mathscr{D i m}_{l f}\left(H_{n}^{9}\right) \leq \sum_{i=1}^{4 n} \frac{1}{4}=n .
$$

Lemma 7. Let $H_{n}^{10}$ be a hexagonal planar circular network for $n \geq 3$ with $n \equiv 1(\bmod 2)$.

(1) Then, for $1 \leq k \leq n,\left|\mathscr{R}_{k}\right|=\left|\mathscr{R}\left\{t_{k} t_{k+1}\right\}\right|=\left|\mathscr{R}\left\{u_{k} v_{k-1}\right\}\right|$ $=\left|\mathscr{R}\left\{u_{k} v_{k}\right\}\right|=\left|\mathscr{R}\left\{s_{k} t_{k+1}\right\}\right|=\left|\mathscr{R}\left\{s_{k} t_{k}\right\}\right|=\left|\mathscr{R}\left\{u_{k} u_{k+1}\right\}\right|$ $=2 n+2$, with $t_{n+1}=t_{1}, \quad v_{n}=v_{0}, \quad u_{n+1}=u_{1}$, and $\left|\cup_{k=1}^{n} \mathscr{R}_{k}\right|=4 n$.

(2) $\left|\mathscr{R}_{k}\right|<\left|\mathscr{R}\left\{t_{k} u_{k}\right\}\right|$ and $\left|\mathscr{R}\left\{t_{k} u_{k}\right\} \cap \cup_{k=1}^{n} \mathscr{R}_{k}\right| \geq\left|\mathscr{R}_{k}\right|$.

Theorem 8. If $H_{n}^{10}$ is a hexagonal planar circular network for $n \geq 3$ with $n \equiv 1(\bmod 2)$, then $\mathscr{D i m}_{l f}\left(H_{n}^{10}\right) \leq(2 n / n+1)$.

Lemma 8. Let $H_{n}^{11}$ be a hexagonal planar circular network for $n \geq 3$ with $n \equiv 1(\bmod 2)$.

(1) Then, for $1 \leq k \leq n, \quad\left|\mathscr{R}_{k}\right|=\left|\mathscr{R}\left\{t_{k} u_{k}\right\}\right|=2 n$, and $\left|\cup_{k=1}^{n} \mathscr{R}_{k}\right|=4 n$.

(2) $\left|\mathscr{R}_{k}\right|<\left|\mathscr{R}\left\{u_{k} v_{k-1}\right\}\right|$ and $\left|\mathscr{R}\left\{u_{k} v_{k-1}\right\} \cap \cup_{k=1}^{n} \mathscr{R}_{k}\right|$ $\geq\left|\mathscr{R}_{k}\right|$ with $v_{n}=v_{0}$.

(3) $\left|\mathscr{R}_{k}\right|<\left|\mathscr{R}\left\{u_{k} v_{k}\right\}\right|$ and $\left|\mathscr{R}\left\{u_{k} v_{k}\right\} \cap \cup_{k=1}^{n} \mathscr{R}_{k}\right| \geq\left|\mathscr{R}_{k}\right|$.

(4) $\left|\mathscr{R}_{k}\right|<\left|\mathscr{R}\left\{s_{k} t_{k+1}\right\}\right|$ and $\left|\mathscr{R}\left\{s_{k} t_{k+1}\right\} \cap \cup_{k=1}^{n} \mathscr{R}_{k}\right| \geq\left|\mathscr{R}_{k}\right|$, with $t_{k+1}=t_{1}$.

(5) $\left|\mathscr{R}_{k}\right|<\left|\mathscr{R}\left\{s_{k} t_{k}\right\}\right|$ and $\left|\mathscr{R}\left\{s_{k} t_{k}\right\} \cap \cup_{k=1}^{n} \mathscr{R}_{k}\right| \geq\left|\mathscr{R}_{k}\right|$.

(6) $\left|\mathscr{R}_{k}\right|<\left|\mathscr{R}\left\{t_{k} v_{k}\right\}\right|$ and $\left|\mathscr{R}\left\{t_{k} v_{k}\right\} \cap \cup_{k=1}^{n} \mathscr{R}_{k}\right| \geq\left|\mathscr{R}_{k}\right|$.

(7) $\left|\mathscr{R}_{k}\right|<\left|\mathscr{R}\left\{s_{k} u_{k+1}\right\}\right|$ and $\left|\mathscr{R}\left\{s_{k} u_{k+1}\right\} \cap \cup_{k=1}^{n} \mathscr{R}_{k}\right| \geq$ $\left|\mathscr{R}_{k}\right|$, with $u_{k+1}=u_{1}$.

Theorem 9. If $H_{n}^{11}$ is a hexagonal planar circular network for $n \geq 3$ with $n \equiv 1(\bmod 2)$, then $\mathscr{D i m}_{l f}\left(H_{n}^{11}\right) \leq 2$.

3.3. LFMD of Rotationally Symmetric Hexagonal Planar Networks with 5 Faces. In this section, we find the sequences of upper bounds of local fractional metric dimension of rotationally symmetric hexagonal planar networks with 5 faces.

Lemma 9. Let $H_{n}^{12}$ be a hexagonal planar circular network for $n \geq 5$ with $n \equiv 1(\bmod 2)$.

(1) Then for $1 \leq k \leq n,\left|\mathscr{R}_{k}\right|=\left|\mathscr{R}\left\{s_{k} t_{k}\right\}\right|=\left|\mathscr{R}\left\{s_{k} t_{k+1}\right\}\right|=$ $\left|\mathscr{R}\left\{u_{k} v_{k}\right\}\right|=\left|\mathscr{R}\left\{u_{k} v_{k-1}\right\}\right|=\left|\mathscr{R}\left\{s_{k} u_{k}\right\}\right|=\left|\mathscr{R}\left\{s_{k} u_{k+1}\right\}\right|$ $=2(n+1)$, with $u_{n+1}=u_{1}, t_{n+1}=t_{1}, v_{n}=v_{0}$, and $\left|\cup_{k=1}^{n} \mathscr{R}_{k}\right|=4 n$.
(2) $\left|\mathscr{R}_{k}\right|<\left|\mathscr{R}\left\{t_{k} u_{k}\right\}\right|$ and $\left|\mathscr{R}\left\{t_{k} u_{k} \cap \cup_{k=1}^{n} \mathscr{R}_{k}\right\}\right| \geq\left|\mathscr{R}_{k}\right|$.

(3) $\left|\mathscr{R}_{k}\right|<\left|\mathscr{R}\left\{u_{k} u_{k+1}\right\}\right|$ and $\left|\mathscr{R}\left\{u_{k} v_{k} \cap \cup_{k=1}^{n} \mathscr{R}_{k}\right\}\right| \geq\left|\mathscr{R}_{k}\right|$, with $u_{n+1}=u_{1}$.

Proof

(1) The set of local resolving neighborhoods of $s_{k} t_{k}$, $s_{k} t_{k+1}, u_{k} v_{k}, u_{k} v_{k-1}, s_{k} u_{k}$, and $s_{k} u_{k+1}$ with $u_{k+1}=$ $u_{1}, t_{k+1}=t_{1}$ is $\mathscr{R}\left\{s_{k} t_{k}\right\}=V\left(H_{n}^{12}\right)-\left\{s_{r} \mid r \equiv k+1+\right.$ $(n-1 / 2), k+2+(n-1 / 2), \ldots, k+n-2(\bmod n)\} \bigcup$ $\left\{t_{r} \mid r \equiv k+1+(n-1 / 2), k+2+(n-1 / 2), \ldots, k+n\right.$ $-2(\bmod n)\} \bigcup\left\{u_{r} \mid r \equiv k(\bmod n), r \equiv k+1+(n-\right.$ $1 / 2), k+2+(n-1 / 2), \ldots, k+n-1(\bmod n)\} \bigcup\left\{v_{r} \mid\right.$ $r \equiv k(\bmod n), r \equiv k+1+(n-1 / 2), k+2+(n-1 /$ $2), \ldots, k+n-1(\bmod n)\}, \quad \mathscr{R}\left\{s_{k} t_{k+1}\right\}=V\left(H_{n}^{12}\right)-$ $\left\{s_{r} \mid r \equiv k+2, k+3, \ldots, k+(n-1 / 2)(\bmod n)\right\} \bigcup^{n}\left\{t_{r} \mid\right.$ $r \equiv k+3, k+4, \ldots, k+(n+1 / 2) \quad(\bmod n)\} \bigcup\left\{u_{r} \mid r\right.$ $\equiv k+1, k+2, \ldots, k+\quad(n+1 / 2)(\bmod n)\} \bigcup\left\{v_{r} \mid r \equiv\right.$ $k, k+1, \ldots, k+(n-1 / 2)(\bmod n)\}, \quad \mathscr{R}\left\{u_{k} v_{k}\right\}=V$ $\left(H_{n}^{12}\right)-\left\{s_{r} \mid r \equiv k+1, k+2, \ldots, k+(n-1 / 2)(\bmod \right.$ $n)\} \bigcup\left\{t_{r} \mid r \equiv k+1, k+2, \ldots, k+(n-1 / 2)(\bmod n)\right\}$ $\bigcup\left\{u_{r} \mid r \equiv k+1, k+2, \ldots, k+(n-1 / 2)(\bmod n)\right\} \bigcup$ $\left\{v_{r} \mid r \equiv k+1, k+2, \ldots, k+(n-1 / 2)(\bmod n)\right\}, \mathscr{R}$ $\left\{u_{k} v_{k-1}\right\}=V\left(H_{n}^{12}\right)-\left\{s_{r} \mid r \equiv k+(n-1 / 2), k+1+(n\right.$ $-1 / 2), \ldots, k+n-2(\bmod n)\} \bigcup\left\{t_{r} \mid r \equiv k+1+(n-1\right.$ /2), $k+2+(n-1 / 2), \ldots, k+n-1(\bmod n)\} \bigcup\left\{u_{r} \mid r\right.$ $\equiv k+1+(n-1 / 2), k+2+(n / 2), \ldots, k+n-1(\bmod$ $n)\} \bigcup\left\{v_{r} \mid r \equiv k+(n-1 / 2), k+1+(n-1 / 2), \ldots, k+\right.$ $n-2(\bmod n)\}, \quad \mathscr{R}\left\{s_{k} u_{k}\right\}=V\left(H_{n}^{12}\right)-\left\{s_{r} \mid r \equiv k+1, k\right.$ $+2, \ldots, k+(n-1 / 2)(\bmod n)\} \bigcup\left\{t_{r} \mid r \equiv k(\bmod n), r \equiv\right.$ $k+2, k+3, \ldots, k+(n-1 / 2)(\bmod n)\} \bigcup\left\{u_{r} \quad \mid r \equiv k+1\right.$, $k+2, \ldots, k+(n-1 / 2)(\bmod n)\} \bigcup\left\{v_{r} \mid r \equiv k+1, k+2\right.$, $\ldots, k+(n-1 / 2)(\bmod n)\}$, and $\mathscr{R}\left\{s_{k} u_{k+1}\right\}=V\left(H_{n}^{12}\right)$ $-\left\{s_{r} \mid r \equiv k+1+(n-1 / 2), k+2+(n-1 / 2), \ldots, k+n-1\right.$ $(\bmod n)\} \bigcup\left\{t_{r} \mid r \equiv k+1(\bmod n), r \equiv k+2+(n-1 / 2)\right.$, $k+3+(n-1 / 2), \ldots, k+n-1(\bmod n)\} \bigcup\left\{u_{r} \mid r \equiv k\right.$ $(\bmod n), r \equiv k+2+(n-1 / 2), k+3+(n-1 / 2), \ldots, k+$ $n-1(\bmod n)\} \bigcup\left\{v_{r} \mid r \equiv k+1+(n-1 / 2), k+2+(n-\right.$ $1 / 2), \ldots, k+n-1 \quad(\bmod n)\}$. Clearly, $\cup_{k=1}^{n} \mathscr{R}_{k}=V$ $\left(H_{n}^{12}\right)$. Therefore, $\left|\cup_{k=1}^{n} \mathscr{R}_{k}\right|=4 n$.

(2) The set of local resolving neighborhoods of $t_{k} u_{k}$ is $\mathscr{R}\left\{t_{k} u_{k}\right\}=V\left(H_{n}^{12}\right)-\left\{s_{r} \mid r \equiv k(\bmod n), r \equiv k+n-1\right.$ $(\bmod n)\} \cup\left\{t_{r} \mid r \equiv k+1(\bmod n), \quad r \equiv k+n-1\right.$ $(\bmod n)\}$. Hence, $\left|\mathscr{R}\left\{t_{k} u_{k}\right\}\right|=4(n-1)$. Since $\left|\mathscr{R}_{k}\right|=2(n+1)<\left|\mathscr{R}\left\{t_{k} u_{k}\right\}\right|=4(n-1), \quad \mid \mathscr{R}\left\{t_{k} u_{k}\right\} \cap$ $\cup_{k=1}^{n} \mathscr{R}_{k}|=4(n-1) \geq| \mathscr{R}_{k} \mid$.

(3) The set of local resolving neighborhoods of $u_{k} u_{k+1}$ with $u_{n+1}=u_{1}$ is $\mathscr{R}\left\{u_{k} u_{k+1}\right\}=V\left(H_{n}^{12}\right)-\left\{s_{r} \mid r \equiv k\right.$ $(\bmod n)\} \bigcup\left\{t_{r} \mid r \equiv k+(n+1 / 2) \quad(\bmod n)\right\} \bigcup\left\{u_{r} \mid r\right.$ $\equiv k+(n+1 / 2)(\bmod n)\} \quad \bigcup\left\{v_{r} \mid r \equiv k(\bmod n)\right\}$. Hence, $\left|\mathscr{R}\left\{u_{k} u_{k+1}\right\}\right|=4(n-1)$. Since $\left|\mathscr{R}_{k}\right|=2(n$ $+1)<\left|\mathscr{R}\left\{u_{k} u_{k+1}\right\}\right|=4(n-1), \quad \mid \mathscr{R}\left\{u_{k} u_{k+1}\right\} \cap \cup_{k=1}^{n}$ $\mathscr{R}_{k}|=4(n-1) \geq| \mathscr{R}_{k} \mid$.

Theorem 10. If $H_{n}^{12}$ is a hexagonal planar circular network with $n \equiv 1(\bmod 2)$, then 


$$
\mathscr{D i t m}_{l f}\left(H_{n}^{12}\right) \leq \begin{cases}\frac{3}{2}, & n=3, \\ \frac{2 n}{n+1}, & n \geq 5 .\end{cases}
$$

Proof. The local resolving neighborhoods are

Case (a): for $n=3,1 \leq k \leq 3$, by Lemma 9 , we have

$$
\begin{aligned}
\overline{R_{1}}= & \mathscr{R}\left\{s_{k} t_{k}\right\}=V\left(H_{3}^{12}\right)-\left\{u_{r} \mid r \equiv k, k+2(\bmod 3)\right\} \cup\left\{v_{r} \mid r \equiv k, k+2(\bmod 3)\right\}, \\
\overline{R_{2}}= & \mathscr{R}\left\{s_{k} t_{k+1}\right\}=V\left(H_{3}^{12}\right)-\left\{u_{r} \mid r \equiv k+1, k+2(\bmod 3)\right\} \cup\left\{v_{r} \mid r \equiv k, k+1(\bmod 3)\right\}, \\
\overline{R_{3}}= & \mathscr{R}\left\{u_{k} v_{k}\right\}=V\left(H_{3}^{12}\right)-\left\{s_{r} \mid r \equiv k+1(\bmod 3)\right\} \cup\left\{t_{r} \mid r \equiv k+1(\bmod 3)\right\} \cup \\
& \cdot\left\{u_{r} \mid r \equiv k+1(\bmod 3)\right\} \cup\left\{v_{r} \mid r \equiv k+1(\bmod 3)\right\}, \\
\overline{R_{4}}= & \mathscr{R}\left\{u_{k} v_{k-1}\right\}=V\left(H_{3}^{12}\right)-\left\{s_{r} \mid r \equiv k+1(\bmod 3)\right\} \cup\left\{t_{r} \mid r \equiv k+2(\bmod 3)\right\} \cup \\
& \cdot\left\{u_{r} \mid r \equiv k+2(\bmod 3)\right\} \cup\left\{v_{r} \mid r \equiv k+1(\bmod 3)\right\}, \\
\overline{R_{5}}= & \mathscr{R}\left\{s_{k} u_{k}\right\}=V\left(H_{3}^{12}\right)-\left\{s_{r} \mid r \equiv k+1(\bmod 3)\right\} \cup\left\{t_{r} \mid r \equiv k(\bmod 3)\right\} \cup\left\{u_{r} \mid r \equiv k+1(\bmod 3)\right\} \\
& \cup\left\{v_{r} \mid r \equiv k+1(\bmod 3)\right\}, \\
\overline{R_{6}}= & \mathscr{R}\left\{s_{k} u_{k+1}\right\}=V\left(H_{3}^{12}\right)-\left\{s_{r} \mid r \equiv k+2(\bmod 3)\right\} \cup\left\{t_{r} \mid r \equiv k+1(\bmod 3)\right\} \cup \\
& \cdot\left\{u_{r} \mid r \equiv k(\bmod 3)\right\} \cup\left\{v_{r} \mid r \equiv k+2(\bmod 3)\right\}, \\
R_{1}= & \mathscr{R}\left\{t_{k} u_{k}\right\}=V\left(H_{3}^{12}\right)-\left\{s_{r} \mid r \equiv k, k+2(\bmod 3)\right\} \cup\left\{t_{r} \mid r \equiv k+1, k+2(\bmod 3)\right\}, \\
R_{2}= & \mathscr{R}\left\{u_{k} u_{k+1}\right\}=V\left(H_{3}^{12}\right)-\left\{s_{r} \mid r \equiv k(\bmod 3)\right\} \cup\left\{t_{r} \mid r \equiv k+2(\bmod 3)\right\} \cup \\
& \cdot\left\{u_{r} \mid r \equiv k+2(\bmod 3)\right\} \cup\left\{v_{r} \mid r \equiv k(\bmod 3)\right\} .
\end{aligned}
$$

The cardinality of each of the local resolving neighborhoods $\overline{R_{1}}, \overline{R_{2}}, \overline{R_{3}}, \overline{R_{4}}, \overline{R_{5}}, \overline{R_{6}}, R_{1}$, and $R_{2}$ for each $k=$ $1,2,3$ is $8,8,8,8,8,8$, and 8 , respectively. Now, we construct a mapping $9: V\left(H_{3}^{12}\right) \longrightarrow[0,1]$ as

$$
\begin{aligned}
\vartheta\left(s_{k}\right) & =\vartheta\left(u_{k}\right)=\vartheta\left(v_{k}\right)=\vartheta\left(t_{k}\right)=\mathcal{\vartheta}\left(u_{k+1}\right) \\
& =\vartheta\left(t_{k+1}\right)=\mathcal{\vartheta}\left(v_{k-1}\right)=\frac{1}{8} .
\end{aligned}
$$

Since 8 is a minimal cardinal number, there must exist a minimal resolving function $\vartheta^{\prime}$ such that $\left|\vartheta^{\prime}\right| \leq|\vartheta|$. Hence,

$$
\mathscr{D i m}_{l f}\left(H_{3}^{12}\right) \leq \sum_{i=1}^{12} \frac{1}{8}=\frac{3}{2} .
$$

Case (b): for $n \geq 5,1 \leq k \leq n$, by Lemma 9 , we have

$$
\begin{aligned}
\overline{R_{1}}= & \mathscr{R}\left\{s_{k} t_{k}\right\}=V\left(H_{n}^{12}\right)-\left\{s_{r} \mid r \equiv k+1+\frac{n-1}{2}, k+2+\frac{n-1}{2}, \ldots, k+n-2(\bmod n)\right\} \cup \\
& \cdot\left\{t_{r} \mid r \equiv k+1+\frac{n-1}{2}, k+2+\frac{n-1}{2}, \ldots, k+n-2(\bmod n)\right\} \cup \\
& \cdot\left\{u_{r} \mid r \equiv k(\bmod n), r \equiv k+1+\frac{n-1}{2}, k+2+\frac{n-1}{2}, \ldots, k+n-1(\bmod n)\right\} \cup \\
& \cdot\left\{v_{r} \mid r \equiv k(\bmod n), r \equiv k+1+\frac{n-1}{2}, k+2+\frac{n-1}{2}, \ldots, k+n-1(\bmod n)\right\},
\end{aligned}
$$




$$
\begin{aligned}
& \overline{R_{2}}=\mathscr{R}\left\{s_{k} t_{k+1}\right\}=V\left(H_{n}^{12}\right)-\left\{s_{r} \mid r \equiv k+2, k+3, \ldots, k+\frac{n-1}{2}(\bmod n)\right\} \cup \\
& \cdot\left\{t_{r} \mid r \equiv k+3, k+4, \ldots, k+\frac{n+1}{2}(\bmod n)\right\} \cup\left\{u_{r} \mid r \equiv k+1, k+2, \ldots, k+\frac{n+1}{2}(\bmod n)\right\} \\
& \cup\left\{v_{r} \mid r \equiv k, k+1, \ldots, k+\frac{n-1}{2}(\bmod n)\right\} \\
& \overline{R_{3}}=\mathscr{R}\left\{u_{k} v_{k}\right\}=V\left(H_{n}^{12}\right)-\left\{s_{r} \mid r \equiv k+1, k+2, \ldots, k+\frac{n-1}{2}(\bmod n)\right\} \cup \\
& \cdot\left\{t_{r} \mid r \equiv k+1, k+2, \ldots, k+\frac{n-1}{2}(\bmod n)\right\} \cup\left\{u_{r} \mid r \equiv k+1, k+2, \ldots, k+\frac{n-1}{2}(\bmod n)\right\} \\
& \cup\left\{v_{r} \mid r \equiv k+1, k+2, \ldots, k+\frac{n-1}{2}(\bmod n)\right\}, \\
& \overline{R_{4}}=\mathscr{R}\left\{u_{k} v_{k-1}\right\}=V\left(H_{n}^{12}\right)-\left\{s_{r} \mid r \equiv k+\frac{n-1}{2}, k+1+\frac{n-1}{2}, \ldots, k+n-2(\bmod n)\right\} \cup \\
& \cdot\left\{t_{r} \mid r \equiv k+1+\frac{n-1}{2}, k+2+\frac{n-1}{2}, \ldots, k+n-1(\bmod n)\right\} \cup \\
& \cdot\left\{u_{r} \mid r \equiv k+1+\frac{n-1}{2}, k+2+\frac{n}{2}, \ldots, k+n-1(\bmod n)\right\} \cup \\
& \left\{v_{r} \mid r \equiv k+\frac{n-1}{2}, k+1+\frac{n-1}{2}, \ldots, k+n-2(\bmod n)\right\}, \\
& \overline{R_{5}}=\mathscr{R}\left\{s_{k} u_{k}\right\}=V\left(H_{n}^{12}\right)-\left\{s_{r} \mid r \equiv k+1, k+2, \ldots, k+\frac{n-1}{2}(\bmod n)\right\} U \\
& \cdot\left\{t_{r} \mid r \equiv k(\bmod n), r \equiv k+2, k+3, \ldots, k+\frac{n-1}{2}(\bmod n)\right\} \cup\left\{u_{r} \mid r \equiv k+1, k+2, \ldots, k+\frac{n-1}{2}(\bmod n)\right\} \\
& \cup\left\{v_{r} \mid r \equiv k+1, k+2, \ldots, k+\frac{n-1}{2}(\bmod n)\right\}, \\
& \overline{R_{6}}=\mathscr{R}\left\{s_{k} u_{k+1}\right\}=V\left(H_{n}^{12}\right)-\left\{s_{r} \mid r \equiv k+1+\frac{n-1}{2}, k+2+\frac{n-1}{2}, \ldots, k+n-1(\bmod n)\right\} \cup \\
& \cdot\left\{t_{r} \mid r \equiv k+1(\bmod n), r \equiv k+2+\frac{n-1}{2}, k+3+\frac{n-1}{2}, \ldots, k+n-1(\bmod n)\right\} \cup \\
& \cdot\left\{u_{r} \mid r \equiv k(\bmod n), r \equiv k+2+\frac{n-1}{2}, k+3+\frac{n-1}{2}, \ldots, k+n-1(\bmod n)\right\} U \\
& \cdot\left\{v_{r} \mid r \equiv k+1+\frac{n-1}{2}, k+2+\frac{n-1}{2}, \ldots, k+n-1(\bmod n)\right\}, \\
& R_{1}=\mathscr{R}\left\{t_{k} u_{k}\right\}=V\left(H_{n}^{12}\right)-\left\{s_{r} \mid r \equiv k(\bmod n), r \equiv k+n-1(\bmod n)\right\} \cup \\
& \cdot\left\{t_{r} \mid r \equiv k+1(\bmod n), r \equiv k+n-1(\bmod n)\right\}, \\
& R_{2}=\mathscr{R}\left\{u_{k} u_{k+1}\right\}=V\left(H_{n}^{12}\right)-\left\{s_{r} \mid r \equiv k(\bmod n)\right\} \cup\left\{t_{r} \mid r \equiv k+\frac{n+1}{2}(\bmod n)\right\} \cup \\
& \cdot\left\{u_{r} \mid r \equiv k+\frac{n+1}{2}(\bmod n)\right\} \cup\left\{v_{r} \mid r \equiv k(\bmod n)\right\} .
\end{aligned}
$$

The cardinality of each of the local resolving neighborhoods $\overline{R_{1}}, \overline{R_{2}}, \overline{R_{3}}, \overline{R_{4}}, \overline{R_{5}}, \overline{R_{6}}, R_{1}$, and $R_{2}$ for each
$1 \leq k \leq n$ is $2(n+1), 2(n+1), 2(n+1), 2(n+1), 2(n+$ $1), 2(n+1), 4(n-1)$, and $4(n-1)$, respectively. Since 
cardinality of $\overline{R_{i}}, 1 \leq i \leq 6$ is $2(n+1)$ which is less than the cardinality of all other resolving neighborhoods and $\cup_{i=1}^{2} R_{i}=V\left(H_{n}^{12}\right)$, this implies that $\left|\overline{R_{j}} \cap \cup_{i=1}^{2} R_{i}\right| \geq\left|R_{i}\right|$. Now, we construct a mapping $9: V\left(H_{n}^{12}\right) \stackrel{\longrightarrow}{\longrightarrow}[0,1]$ as

$$
\begin{aligned}
\vartheta\left(s_{k}\right) & =\vartheta\left(u_{k}\right)=\vartheta\left(v_{k}\right)=\vartheta\left(t_{k}\right)=\vartheta\left(u_{k+1}\right) \\
& =\vartheta\left(t_{k+1}\right)=\vartheta\left(v_{k-1}\right)=\frac{1}{2(n+1)} .
\end{aligned}
$$

Since $2(n+1)$ is a minimal cardinal number, there must exist a minimal resolving function $\vartheta^{\prime}$ such that $\left|\vartheta^{\prime}\right| \leq|\vartheta|$. Hence,

$$
\mathscr{D i m}_{l f}\left(H_{n}^{12}\right) \leq \sum_{i=1}^{4 n} \frac{1}{2(n+1)}=\frac{2 n}{n+1} .
$$

Lemma 10. Let $H_{n}^{13}$ be a hexagonal planar circular network for $n \geq 5$ with $n \equiv 1(\bmod 2)$.

(1) Then, for $1 \leq k \leq n,\left|\mathscr{R}_{k}\right|=\left|\mathscr{R}\left\{t_{k} u_{k}\right\}\right|=\left|\mathscr{R}\left\{t_{k} u_{k+1}\right\}\right|$ $=2(n+1)$, with $u_{1}=u_{n+1}$ and $\left|\cup_{k=1}^{n} \mathscr{R}_{k}\right|=4 n$.

(2) $\left|\mathscr{R}_{k}\right|<\left|\mathscr{R}\left\{s_{k} t_{k}\right\}\right|$ and $\left|\mathscr{R}\left\{k_{k} t_{k}\right\} \cap \cup_{k=1}^{n} \mathscr{R}_{k}\right| \geq\left|\mathscr{R}_{k}\right|$.

(3) $\left|\mathscr{R}_{k}\right|<\left|\mathscr{R}\left\{u_{k} v_{k}\right\}\right|$ and $\left|\mathscr{R}\left\{u_{k} v_{k}\right\} \cap \cup_{k=1}^{n} \mathscr{R}_{k}\right| \geq\left|\mathscr{R}_{k}\right|$.

(4) $\left|\mathscr{R}_{k}\right|<\left|\mathscr{R}\left\{t_{k} t_{k+1}\right\}\right|$ and $\left|\mathscr{R}\left\{t_{k} t_{k+1}\right\} \cap \cup_{k=1}^{n} \mathscr{R}_{k}\right| \geq\left|\mathscr{R}_{k}\right|$, with $t_{n+1}=t_{1}$.

(5) $\left|\mathscr{R}_{k}\right|<\left|\mathscr{R}\left\{u_{k} u_{k+1}\right\}\right|$ and $\left|\mathscr{R}\left\{u_{k} u_{k+1}\right\} \cap \cup_{k=1}^{n} \mathscr{R}_{k}\right| \geq$ $\left|\mathscr{R}_{k}\right|$, with $u_{n+1}=u_{1}$.

(6) $\left|\mathscr{R}_{k}\right|<\left|\mathscr{R}\left\{s_{k} t_{k+1}\right\}\right|$ and $\left|\mathscr{R}\left\{s_{k} t_{k+1}\right\} \cap \cup_{k=1}^{n} \mathscr{R}_{k}\right| \geq\left|\mathscr{R}_{k}\right|$, with $t_{1}=t_{n+1}$.

(7) $\left|\mathscr{R}_{k}\right|<\left|\mathscr{R}\left\{u_{k} v_{k-1}\right\}\right|$ and $\left|\mathscr{R}\left\{u_{k} v_{k-1}\right\} \cap \cup_{k=1}^{n} \mathscr{R}_{k}\right| \geq$ $\left|\mathscr{R}_{k}\right|$, with $v_{0}=v_{n}$.

Theorem 11. If $H_{n}^{13}$ is a hexagonal planar circular network with $n \equiv 1(\bmod 2)$, then

$$
\operatorname{Dim}_{l f}\left(H_{n}^{13}\right) \leq \begin{cases}2, & n=3, \\ \frac{2 n}{n+1}, & n \geq 5\end{cases}
$$

Lemma 11. Let $H_{n}^{14}$ be a hexagonal planar circular network for $n \geq 3$ with $n \equiv 1(\bmod 2)$.

(1) Then, for $1 \leq k \leq n,\left|\mathscr{R}_{k}\right|=\left|\mathscr{R}\left\{t_{k} u_{k}\right\}\right|=\left|\mathscr{R}\left\{s_{k} u_{k+1}\right\}\right|=$ $\left|\mathscr{R}\left\{s_{k} v_{k}\right\}\right|=\left|\mathscr{R}\left\{t_{k} v_{k}\right\}\right|=2 n$, with $u_{1}=u_{n+1}$ and $\left|\cup_{k=1}^{n} \mathscr{R}_{k}\right|=4 n$.

(2) $\left|\mathscr{R}_{k}\right|<\left|\mathscr{R}\left\{u_{k} v_{k}\right\}\right|$ and $\left|\mathscr{R}\left\{u_{k} v_{k}\right\} \cap \cup_{k=1}^{n} \mathscr{R}_{k}\right| \geq\left|\mathscr{R}_{k}\right|$.

(3) $\left|\mathscr{R}_{k}\right|<\left|\mathscr{R}\left\{s_{k} t_{k}\right\}\right|$ and $\left|\mathscr{R}\left\{s_{k} t_{k}\right\} \cap \cup_{k=1}^{n} \mathscr{R}_{k}\right| \geq\left|\mathscr{R}_{k}\right|$.

(4) $\left|\mathscr{R}_{k}\right|<\left|\mathscr{R}\left\{s_{k} t_{k+1}\right\}\right|$ and $\left|\mathscr{R}\left\{s_{k} t_{k+1}\right\} \cap \cup_{k=1}^{n} \mathscr{R}_{k}\right| \geq\left|\mathscr{R}_{k}\right|$, with $t_{1}=t_{n+1}$.

(5) $\left|\mathscr{R}_{k}\right|<\left|\mathscr{R}\left\{u_{k} v_{k-1}\right\}\right|$ and $\left|\mathscr{R}\left\{u_{k} v_{k-1}\right\} \cap \cup_{k=1}^{n} \mathscr{R}_{k}\right| \geq$ $\left|\mathscr{R}_{k}\right|$, with $v_{0}=v_{n}$.
Theorem 12. If $H_{n}^{14}$ is a hexagonal planar circular network for $n \geq 3$ with $n \equiv 1(\bmod 2)$, then $\mathscr{D} \mathbf{i m}_{l f}\left(H_{n}^{14}\right) \leq 2$.

Lemma 12. Let $H_{n}^{15}$ be a hexagonal planar circular network for $n \geq 3$ with $n \equiv 1(\bmod 2)$.

(1) Then, for $1 \leq k \leq n$, $\left|\mathscr{R}_{k}\right|=\left|\mathscr{R}\left\{s_{k} u_{k+1}\right\}\right|=\left|\mathscr{R}\left\{t_{k} v_{k}\right\}\right|=4$ and $\left|\cup_{k=1}^{n} \mathscr{R}_{k}\right|=$ $4 n$ with $u_{n+1}=u_{1}$.

(2) $\left|\mathscr{R}_{k}\right|<\left|\mathscr{R}\left\{s_{k} t_{k}\right\}\right|$ and $\left|\mathscr{R}\left\{s_{k} t_{k}\right\} \cap \cup_{k=1}^{n} \mathscr{R}_{k}\right| \geq\left|\mathscr{R}_{k}\right|$.

(3) $\left|\mathscr{R}_{k}\right|<\left|\mathscr{R}\left\{t_{k} u_{k}\right\}\right|$ and $\left|\mathscr{R}\left\{t_{k} u_{k}\right\} \cap \cup_{k=1}^{n} \mathscr{R}_{k}\right| \geq\left|\mathscr{R}_{k}\right|$.

(4) $\left|\mathscr{R}_{k}\right|<\left|\mathscr{R}\left\{u_{k} v_{k}\right\}\right|$ and $\left|\mathscr{R}\left\{u_{k} v_{k}\right\} \cap \cup_{k=1}^{n} \mathscr{R}_{k}\right| \geq\left|\mathscr{R}_{k}\right|$.

(5) $\left|\mathscr{R}_{k}\right|<\left|\mathscr{R}\left\{t_{k} u_{k+1}\right\}\right|$ and $\left|\mathscr{R}\left\{t_{k} u_{k+1}\right\} \cap \cup_{k=1}^{n} \mathscr{R}_{k}\right| \geq$ $\left|\mathscr{R}_{k}\right|$, with $u_{n+1}=u_{1}$.

(6) $\left|\mathscr{R}_{k}\right|<\left|\mathscr{R}\left\{s_{k} t_{k+1}\right\}\right|$ and $\left|\mathscr{R}\left\{s_{k} t_{k+1}\right\} \cap \cup_{k=1}^{n} \mathscr{R}_{k}\right| \geq\left|\mathscr{R}_{k}\right|$, with $t_{1}=t_{n+1}$.

(7) $\left|\mathscr{R}_{k}\right|<\left|\mathscr{R}\left\{u_{k} v_{k-1}\right\}\right|$ and $\left|\mathscr{R}\left\{u_{k} v_{k-1}\right\} \cap \cup_{k=1}^{n} \mathscr{R}_{k}\right| \geq$ $\left|\mathscr{R}_{k}\right|$ with $v_{0}=v_{n}$.

Proof

(1) The set of local resolving neighborhoods of $s_{k} u_{k+1}$ and $t_{k} v_{k}$ with $u_{n+1}=u_{1}$ is $\mathscr{R}\left\{s_{k} u_{k+1}\right\}=V\left(H_{n}^{15}\right)-\left\{s_{r}\right.$ $\mid r \equiv k+1, k+2, \ldots, k+n-1(\bmod n)\} \cup\left\{t_{r} \mid r \equiv k, k+\right.$ $1, \ldots, k+n-1(\bmod n)\} \cup\left\{u_{r} \mid r \equiv k(\bmod n), r \equiv k+\right.$ $2, k+3, \ldots, k+n-1 \quad(\bmod n)\} \cup\left\{v_{r} \mid r \quad \equiv k+2, k+3\right.$, $\ldots, k+n-1(\bmod n)\}$ and $\mathscr{R}\left\{t_{k} v_{k}\right\}=V\left(H_{n}^{15}\right)-\left\{s_{r} \mid r\right.$ $\equiv k+1, k+2, \ldots, k+n-2(\bmod n)\} \cup\left\{t_{r} \mid r \equiv k+1, k+\right.$ $2, \ldots, k+n-1(\bmod n)\}\left\{u_{r} \mid r \equiv k, k+1, \quad \ldots, k+n-1\right.$ $(\bmod n)\}\left\{v_{r} \mid r \equiv k+1, k+2, \ldots, k+n-1 \quad(\bmod n)\right\}$. Clearly, $\cup_{k=1}^{n} \mathscr{R}_{k}=V\left(H_{n}^{15}\right)$. Therefore, $\left|\cup_{k=1}^{n} \mathscr{R}_{k}\right|=$ $4 n$.

(2) The set of local resolving neighborhoods of $s_{k} t_{k}$ is $\mathscr{R}\left\{s_{k} t_{k}\right\}=V\left(H_{n}^{15}\right)-\left\{u_{r} \mid r \equiv k+1 \quad(\bmod n)\right\} \cup\left\{v_{r} \mid\right.$ $r \equiv k+1(\bmod n)\}$. Hence, $\left|\mathscr{R}\left\{s_{k} t_{k}\right\}\right|=2(2 n-1)$. Since $\left|\mathscr{R}_{k}\right|=4<\left|\mathscr{R}\left\{s_{k} t_{k}\right\}\right|=2(2 n-1), \mid \mathscr{R}\left\{s_{k} t_{k}\right\} \cap$ $\cup_{k=1}^{n} \mathscr{R}_{k}|=2(2 n-1) \geq| \mathscr{R}_{k} \mid$.

(3) The set of local resolving neighborhoods of $t_{k} u_{k}$ is $\mathscr{R}\left\{t_{k} u_{k}\right\}=V\left(H_{n}^{15}\right)-\left\{s_{r} \mid r \equiv k+n-1 \quad(\bmod n)\right\} \cup$ $\left\{v_{r} \mid r \equiv k(\bmod n)\right\}$. Hence, $\left|\mathscr{R}\left\{t_{k} u_{k}\right\}\right|=2(2 n-1)$. Since $\left|\mathscr{R}_{k}\right|=4<\left|\mathscr{R}\left\{t_{k} u_{k}\right\}\right|=2(2 n-1), \mid \mathscr{R}\left\{t_{k} u_{k}\right\} \cap$ $\cup_{k=1}^{n} \mathscr{R}_{k}|=2(2 n-1) \geq| \mathscr{R}_{k} \mid$.

(4) The set of local resolving neighborhoods of $u_{k} v_{k}$ is $\mathscr{R}\left\{u_{k} v_{k}\right\}=V\left(H_{n}^{15}\right)-\left\{s_{r} \mid r \equiv k(\bmod n)\right\} \cup\left\{t_{r} \mid r \equiv k\right.$ $(\bmod n)\}$. Hence, $\left|\mathscr{R}\left\{u_{k} v_{k}\right\}\right|=2(2 n-1)$. Since $\left|\mathscr{R}_{k}\right|=4<\left|\mathscr{R}\left\{u_{k} v_{k}\right\}\right|=2(2 n-1), \quad \mid \mathscr{R}\left\{u_{k} v_{k}\right\} \cap \cup_{k=1}^{n}$ $\mathscr{R}_{k}|=2(2 n-1) \geq| \mathscr{R}_{k} \mid$.

(5) The set of local resolving neighborhoods of $t_{k} u_{k+1}$, with $u_{n+1}=u_{1}$ is $\mathscr{R}\left\{t_{k} u_{k+1}\right\}=V\left(H_{n}^{15}\right)-\left\{s_{r} \mid r \equiv k(\bmod n)\right\}$ $\cup\left\{v_{r} \mid r \equiv k(\bmod n)\right\}$. Hence, $\left|\mathscr{R}\left\{t_{k} u_{k+1}\right\}\right|=2(2 n-1)$. Since $\left|\mathscr{R}_{k}\right|=4<\left|\mathscr{R}\left\{t_{k} u_{k}\right\}\right|=2(2 n-1), \quad \mid \mathscr{R}\left\{t_{k} u_{k}\right\} \cap$ $\cup_{k=1}^{n} \mathscr{R}_{k}|=2(2 n-1) \geq| \mathscr{R}_{k} \mid$.

(6) The set of local resolving neighborhoods of $s_{k} t_{k+1}$ with $t_{n+1}=t_{1}$ is $\mathscr{R}\left\{s_{k} t_{k+1}\right\}=V\left(H_{n}^{15}\right)-\left\{u_{r} \mid r \equiv k+\right.$ $1(\bmod n)\} \cup\left\{v_{r} \mid r \equiv k(\bmod n)\right\}$. Hence, $\left|\mathscr{R}\left\{s_{k} t_{k+1}\right\}\right|$ 
TABLE 1: Sequences of local fractional metric dimension of rotationally symmetric hexagonal planar networks.

\begin{tabular}{|c|c|}
\hline Network & Upper bound of $\mathscr{D} \mathfrak{i} \mathfrak{m}_{l f}$ \\
\hline $\begin{array}{l}H_{n}^{1}, H_{n}^{2}, H_{n}^{5} \\
H_{n}^{3}, H_{n}^{10} H_{n}^{17} \\
H_{n}^{4}\end{array}$ & $\begin{array}{c}1 \\
(2 n / n+1) \\
(4 n / 2 n+1)\end{array}$ \\
\hline$H_{n}^{6}, H_{n}^{11}, H_{n}^{14}$ & 2 \\
\hline$H_{n}^{7}$ & $\begin{array}{ll}2, & n=3 \\
(4 n / 2 n+3), & n \geq 5\end{array}$ \\
\hline$H_{n}^{8}$ & $\begin{cases}2, & n=3 \\
(n / 2), & n \geq 5\end{cases}$ \\
\hline$H_{n}^{9}, H_{n}^{15}, H_{n}^{16}$ & $n$ \\
\hline$H_{n}^{12}$ & $\begin{array}{ll}(3 / 2), & n=3 \\
(2 n / n+1), & n \geq 5\end{array}$ \\
\hline$H_{n}^{13}$ & $\begin{array}{ll}2, & n=3 \\
(2 n / n+1), & n \geq 5\end{array}$ \\
\hline
\end{tabular}

(7) The set of local resolving neighborhoods of $u_{k} v_{k-1}$ with $v_{n-1}=v_{0}$ is $\mathscr{R}\left\{u_{k} v_{k+1}\right\}=V\left(H_{n}^{15}\right)-\left\{s_{r} \mid r \equiv k+\right.$ $n-2(\bmod n)\} \cup\left\{t_{r} \mid r \equiv k+n-1(\bmod n)\right\}$. Hence, $\left|\mathscr{R}\left\{u_{k} v_{k+1}\right\}\right|=2(2 n-1)$. Since $\left|\mathscr{R}_{k}\right|=4<\mid \mathscr{R}\left\{u_{k}\right.$ $\left.v_{k-1}\right\}|=2(2 n-1), \quad| \mathscr{R}\left\{u_{k} v_{k}\right\} \cap \cup_{k=1}^{n} \mathscr{R}_{k} \mid=2(2 n-$ 1) $\geq\left|\mathscr{R}_{k}\right|$.

Theorem 13. If $H_{n}^{15}$ is a hexagonal planar circular network for $n \geq 3$ with $n \equiv 1(\bmod 2)$, then $\mathscr{D} \mathbf{i m}_{l f}\left(H_{n}^{15}\right) \leq n$.

Proof. When $n \geq 3$ with $n \equiv 1(\bmod 2)$, by Lemma 12 , the local resolving neighborhoods are

$=2(2 n-1)$. Since $\left|\mathscr{R}_{k}\right|=4<\left|\mathscr{R}\left\{s_{k} t_{k}\right\}\right|=2(2 n-1)$, $\left|\mathscr{R}\left\{s_{k} t_{k}\right\} \cap \cup_{k=1}^{n} \mathscr{R}_{k}\right|=2(2 n-1) \geq\left|\mathscr{R}_{k}\right|$.

$$
\begin{aligned}
\overline{R_{1}}= & \mathscr{R}\left\{s_{k} u_{k+1}\right\}=V\left(H_{n}^{15}\right)-\left\{s_{r} \mid r \equiv k+1, k+2, \ldots, k+n-1(\bmod n)\right\} \cup\left\{t_{r} \mid r \equiv k, k+1, \ldots, k+n-1(\bmod n)\right\} \\
& \cup\left\{u_{r} \mid r \equiv k(\bmod n), r \equiv k+2, k+3, \ldots, k+n-1(\bmod n)\right\} \cup\left\{v_{r} \mid r \equiv k+2, k+3, \ldots, k+n-1(\bmod n)\right\}, \\
\overline{R_{2}}= & \mathscr{R}\left\{t_{k} v_{k}\right\}=V\left(H_{n}^{15}\right)-\left\{s_{r} \mid r \equiv k+1, k+2, \ldots, k+n-2(\bmod n)\right\} \cup\left\{t_{r} \mid r \equiv k+1, k+2, \ldots, k+n-1(\bmod n)\right\} \\
& \cup\left\{u_{r} \mid r \equiv k, k+1, \ldots, k+n-1(\bmod n)\right\} \cup\left\{v_{r} \mid r \equiv k+1, k+2, \ldots, k+n-1(\bmod n)\right\}, \\
R_{1}= & \mathscr{R}\left\{s_{k} t_{k}\right\}=V\left(H_{n}^{15}\right)-\left\{u_{r} \mid r \equiv k+1(\bmod n)\right\} \cup\left\{v_{r} \mid r \equiv k+1(\bmod n)\right\}, \\
R_{2}= & \mathscr{R}\left\{t_{k} u_{k}\right\}=V\left(H_{n}^{15}\right)-\left\{s_{r} \mid r \equiv k+n-1(\bmod n)\right\} \cup\left\{v_{r} \mid r \equiv k(\bmod n)\right\}, \\
R_{3}= & \mathscr{R}\left\{u_{k} v_{k}\right\}=V\left(H_{n}^{15}\right)-\left\{s_{r} \mid r \equiv k(\bmod n)\right\} \cup\left\{t_{r} \mid r \equiv k(\bmod n)\right\}, \\
R_{4}= & \mathscr{R}\left\{t_{k} u_{k+1}\right\}=V\left(H_{n}^{15}\right)-\left\{s_{r} \mid r \equiv k(\bmod n)\right\} \cup\left\{v_{r} \mid r \equiv k(\bmod n)\right\}, \\
R_{5}= & \mathscr{R}\left\{s_{k} t_{k+1}\right\}=V\left(H_{n}^{15}\right)-\left\{u_{r} \mid r \equiv k+1(\bmod n)\right\} \cup\left\{v_{r} \mid r \equiv k(\bmod n)\right\}, \\
R_{6}= & \mathscr{R}\left\{u_{k} v_{k-1}\right\}=V\left(H_{n}^{15}\right)-\left\{s_{r} \mid r \equiv k+n-2(\bmod n)\right\} \cup\left\{t_{r} \mid r \equiv k+n-1(\bmod n)\right\} .
\end{aligned}
$$

The cardinality of each of the local resolving neighborhoods $\overline{R_{1}}, \overline{R_{2}}, R_{1}, R_{2}, R_{3}, R_{4}, R_{5}$, and $R_{6}$ for each $1 \leq k \leq n$ is 4,4 , $4 n-2,4 n-2,4 n-2,4 n-2,4 n-2,4 n-2$, and $4 n-2$, respectively. Since cardinality of $\overline{R_{i}}, 1 \leq i \leq 2$ is 4 which is less than the cardinality of all other resolving neighborhoods and $\cup_{i=1}^{6} R_{i}=V\left(H_{n}^{15}\right)$, this implies that $\left|\overline{R_{j}} \cap \cup_{i=1}^{6} R_{i}\right| \geq\left|R_{i}\right|$. Now, we construct a mapping $\vartheta: V\left(H_{n}^{15}\right) \longrightarrow[0,1]$ as

$$
\vartheta\left(s_{k}\right)=\vartheta\left(u_{k+1}\right)=\vartheta\left(v_{k}\right)=\vartheta\left(t_{k}\right)=\frac{1}{4} .
$$

Since 4 is a minimal cardinal number, there must exist a minimal resolving function $\vartheta^{\prime}$ such that $\left|\vartheta^{\prime}\right| \leq|\vartheta|$. Hence,

$$
\mathscr{D i m}_{l f}\left(H_{n}^{15}\right) \leq \sum_{i=1}^{4 n} \frac{1}{4}=n .
$$

Lemma 13. Let $H_{n}^{16}$ be a hexagonal planar circular network for $n \geq 3$ with $n \equiv 1(\bmod 2)$.

(1) Then, for $1 \leq k \leq n,\left|\mathscr{R}_{k}\right|=\left|\mathscr{R}\left\{t_{k} u_{k}\right\}\right|=\left|\mathscr{R}\left\{s_{k} v_{k}\right\}\right|=$ 4 and $\left|\cup_{k=1}^{n} \mathscr{R}_{k}\right|=4 n$.

(2) $\left|\mathscr{R}_{k}\right|<\left|\mathscr{R}\left\{s_{k} t_{k}\right\}\right|$ and $\left|\mathscr{R}\left\{s_{k} t_{k}\right\} \cap \cup_{k=1}^{n} \mathscr{R}_{k}\right| \geq\left|\mathscr{R}_{k}\right|$.

(3) $\left|\mathscr{R}_{k}\right|<\left|\mathscr{R}\left\{u_{k} v_{k}\right\}\right|$ and $\left|\mathscr{R}\left\{u_{k} v_{k}\right\} \cap \cup_{k=1}^{n} \mathscr{R}_{k}\right| \geq\left|\mathscr{R}_{k}\right|$.

(4) $\left|\mathscr{R}_{k}\right|<\left|\mathscr{R}\left\{s_{k} u_{k+1}\right\}\right| \quad$ and $\left|\mathscr{R}\left\{s_{k} u_{k+1}\right\} \cap \cup_{k=1}^{n} \mathscr{R}_{k}\right|$ $\geq\left|\mathscr{R}_{k}\right|$, with $u_{n+1}=u_{1}$.

(5) $\left|\mathscr{R}_{k}\right|<\left|\mathscr{R}\left\{s_{k} u_{k}\right\}\right|$ and $\left|\mathscr{R}\left\{s_{k} u_{k}\right\} \cap \cup_{k=1}^{n} \mathscr{R}_{k}\right| \geq\left|\mathscr{R}_{k}\right|$.

(6) $\left|\mathscr{R}_{k}\right|<\left|\mathscr{R}\left\{s_{k} t_{k+1}\right\}\right|$ and $\left|\mathscr{R}\left\{s_{k} t_{k+1}\right\} \cap \cup_{k=1}^{n} \mathscr{R}_{k}\right| \geq\left|\mathscr{R}_{k}\right|$, with $t_{n+1}=t_{1}$.

(7) $\left|\mathscr{R}_{k}\right|<\left|\mathscr{R}\left\{u_{k} v_{k-1}\right\}\right|$ and $\left|\mathscr{R}\left\{u_{k} v_{k-1}\right\} \cap \cup_{k=1}^{n} \mathscr{R}_{k}\right|$ $\geq\left|\mathscr{R}_{k}\right|$, with $v_{n}=v_{0}$. 


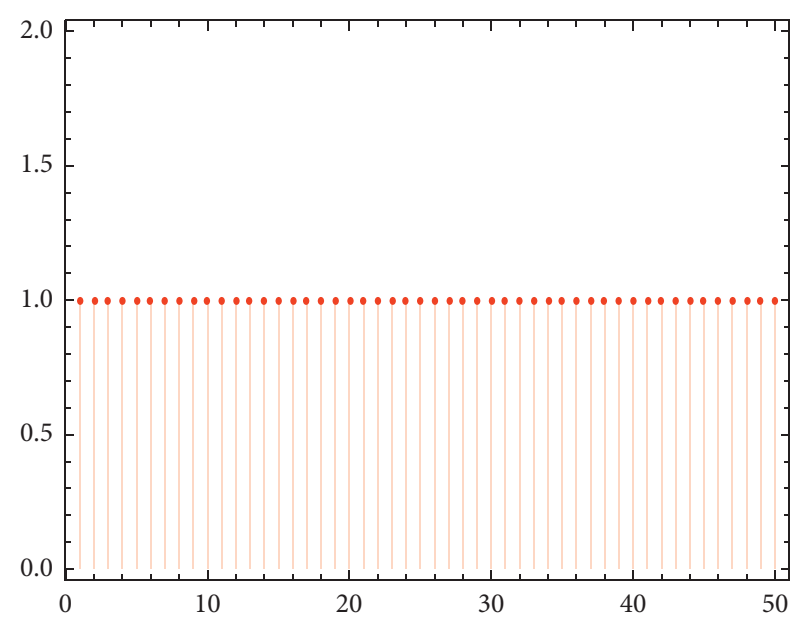

(a)

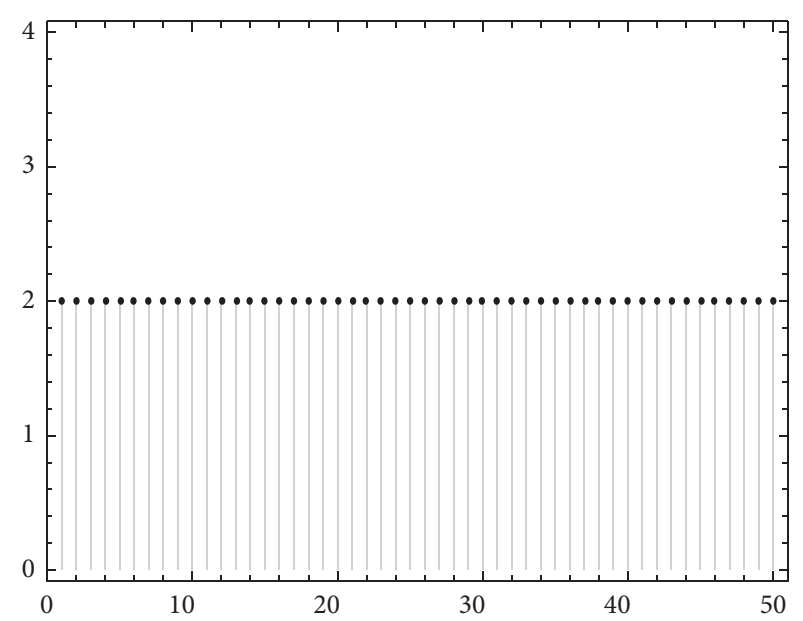

(b)

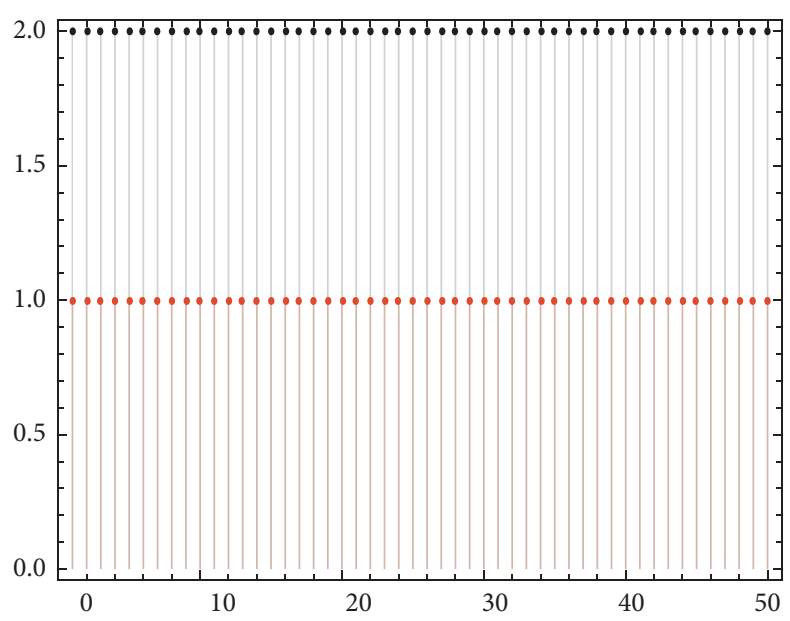

(c)

FIGURE 6: Graph of sequences of rotationally symmetric hexagonal planar networks $H_{n}^{i}, i=1,2,5,6,11,14$, and their comparison.

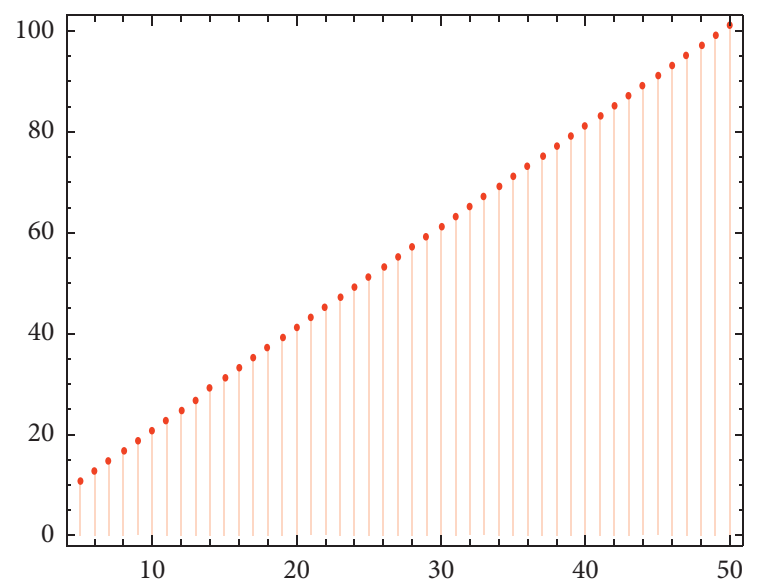

(a)

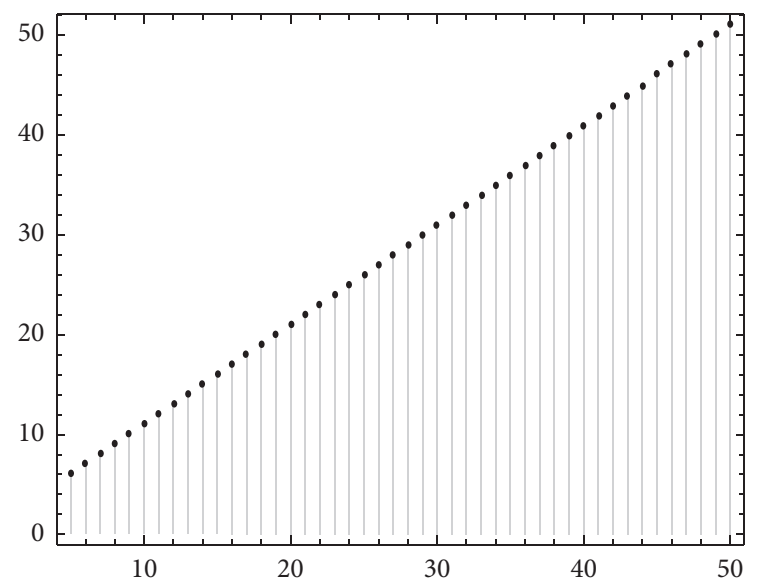

(b)

Figure 7: Continued. 


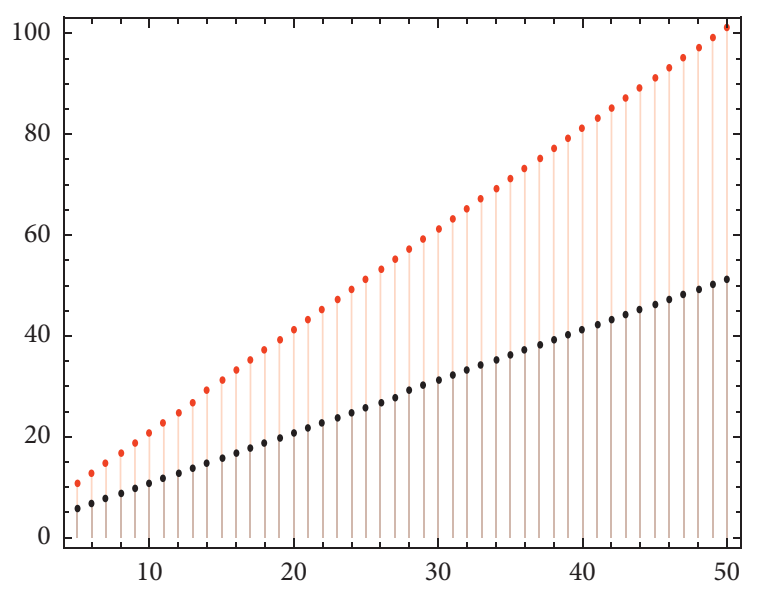

(c)

FIGURE 7: Graph of sequences of rotationally symmetric hexagonal planar networks $H_{n}^{i}, i=8,9,15,16$, and their comparison.

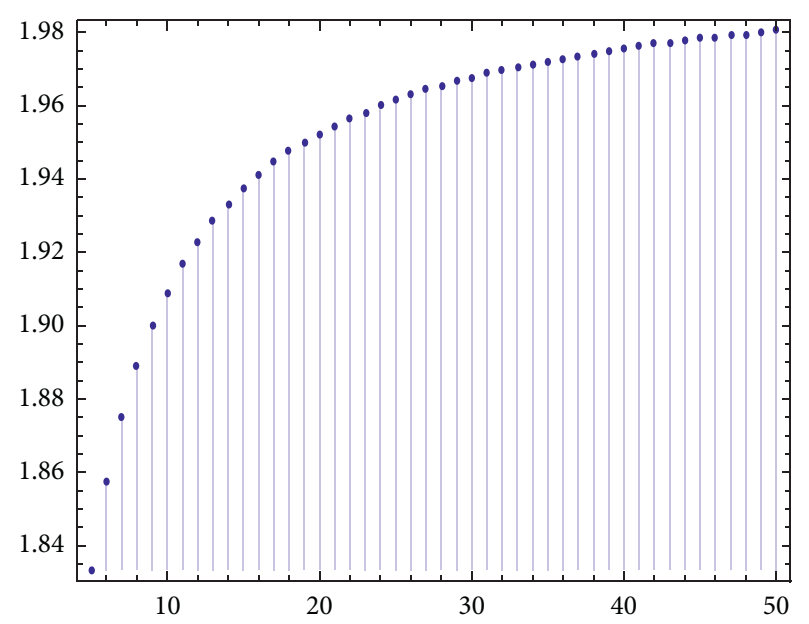

(a)

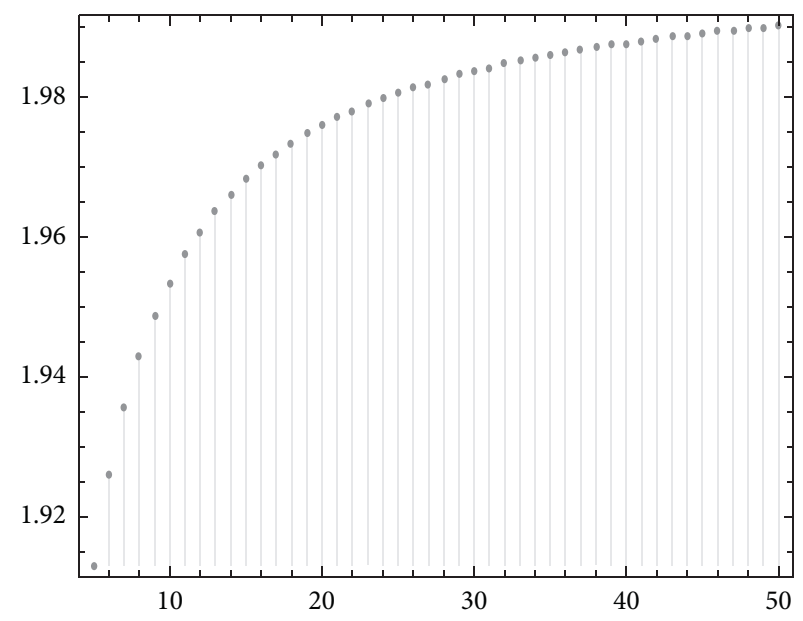

(b)

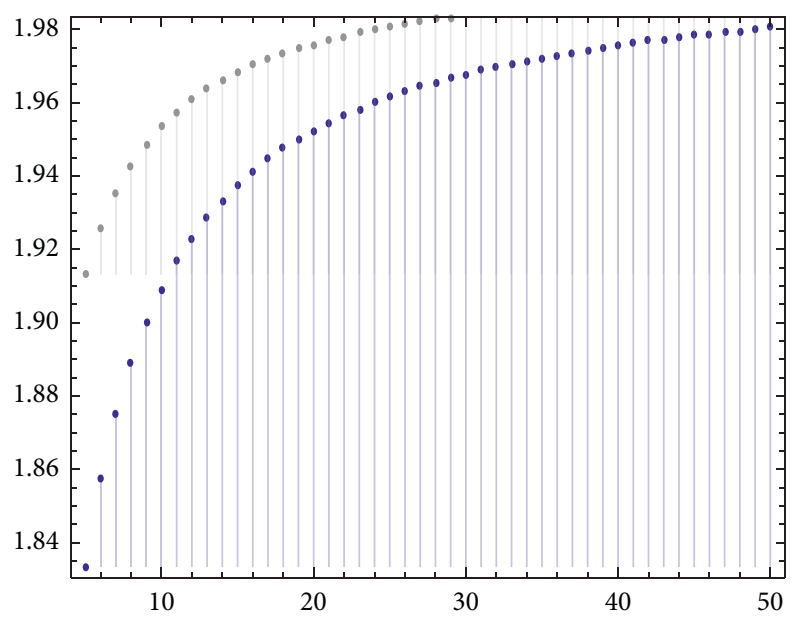

(c)

Figure 8: Graph of sequences of rotationally symmetric hexagonal planar networks $H_{n}^{i}, i=3,4,7,10,12,13,17$, and their comparison. 
Theorem 14. If $H_{n}^{16}$ is a hexagonal planar circular network for $n \geq 3$ with $n \equiv 1(\bmod 2)$, then $\mathscr{D} \mathbf{i m}_{l f}\left(H_{n}^{16}\right) \leq n$.

Lemma 14. Let $H_{n}^{17}$ be a hexagonal planar circular network for $n \geq 3$ with $n \equiv 1(\bmod 2)$.

(1) Then, for $1 \leq k \leq n,\left|\mathscr{R}_{k}\right|=\left|\mathscr{R}\left\{s_{k} t_{k}\right\}\right|=\left|\mathscr{R}\left\{s_{k} t_{k+1}\right\}\right|=$ $\left|\mathscr{R}\left\{t_{k} u_{k}\right\}\right|=\left|\mathscr{R}\left\{u_{k} v_{k}\right\}\right|=\left|\mathscr{R}\left\{u_{k} v_{k-1}\right\}\right|=\left|\mathscr{R}\left\{t_{k+1} u_{k}\right\}\right|$ $=2(n+1)$, with $t_{1}=t_{n+1}, u_{1}=u_{n+1}, v_{0}=v_{n}$, and $\left|\cup_{k=1}^{n} \mathscr{R}_{k}\right|=4 n$.

(2) $\left|\mathscr{R}_{k}\right|<\left|\mathscr{R}\left\{t_{k} t_{k+1}\right\}\right|$ and $\left|\mathscr{R}\left\{t_{k} t_{k+1}\right\} \cap \cup_{k=1}^{n} \mathscr{R}_{k}\right| \geq\left|\mathscr{R}_{k}\right|$ with $t_{n+1}=t_{1}$.

(3) $\left|\mathscr{R}_{k}\right|<\left|\mathscr{R}\left\{v_{k} t_{k+1}\right\}\right|$ and $\left|\mathscr{R}\left\{v_{k} t_{k+1}\right\} \cap \cup_{k=1}^{n} \mathscr{R}_{k}\right| \geq\left|\mathscr{R}_{k}\right|$ with $t_{n+1}=t_{1}$.

Theorem 15. If $H_{n}^{17}$ is a hexagonal planar circular network for $n \geq 3$ with $n \equiv 1(\bmod 2)$, then $\mathscr{D i m}_{l f}\left(H_{n}^{17}\right) \leq(2 n / n+1)$.

\section{Table of Sequences of LFMD of Rotationally Symmetric Hexagonal Planar Networks and Their Comparison via Graphs}

In this section, we propose the table of sequences of local fractional metric dimension of rotationally symmetric hexagonal planar networks and their comparison via plotting the graphs of these sequences (Table 1).

The boundedness comparison of sequences of local farctional metric dimension of rotationally symmetric hexagonal planar networks are shown in Figures 6-8.

\section{Conclusion}

In this paper, we discuss the local fractional metric dimension of rotationally symmetric hexagonal planar networks. The sequences of their upper bounds are investigated. The sequences of upper bounds of each rotationally symmetric hexagonal planar network are plotted for compression of their bounds. We can see easily that there are two types of sequences: bounded as well as unbounded. In future work, researchers can find domination and spectral number of rotationally symmetric hexagonal planar networks.

\section{Data Availability}

No data were used to support this study.

\section{Conflicts of Interest}

The authors declare that they have no conflicts of interest.

\section{Acknowledgments}

The authors extend their appreciation to the Deanship of Scientific Research at King Saud University for funding this work through research group no. RG-1440-010.

\section{References}

[1] G. Chartrand and L. Lesniak, Graphs \& Digraphs, CRC Press, Boca Raton, FL, USA, 4th edition, 2005.

[2] J. L. Gross and J. Yellen, Graph Theory and its Applications, CRC Press, Boca Raton, FL, USA, 2nd edition, 2005.

[3] D. B. West, Introduction to Graph Theory, Prentice-Hall, Upper Saddle River, NJ, USA, 2001.

[4] G. Chartrand, L. Eroh, M. A. Johnson, and O. R. Oellermann, "Resolvability in graphs and the metric dimension of a graph," Discrete Applied Mathematics, vol. 105, no. 1-3, pp. 99-113, 2000.

[5] P. S. Buczkowski, G. Chartrand, C. Poisson, and P. Zhang, "On k- dimensional graphs and their bases," Periodica Mathematica Hungarica, vol. 46, no. 1, pp. 9-15, 2003.

[6] P. J. Slater, "Dominating and reference sets in a graph," Journal of Mathematical and Physical Sciences, vol. 22, no. 4, pp. 445-455, 1988.

[7] P. J. Slater, "Domination and location in acyclic graphs," Networks, vol. 17, no. 1, pp. 55-64, 1987.

[8] P. J. Slater, "Leaves of trees," Congressus Numerantium, vol. 14, pp. 549-559, 1975.

[9] F. Harary and R. A. Melter, "On the metric dimension of a graph,” Ars Combinatoria, vol. 2, pp. 191-195, 1976.

[10] I. Javaid, M. T. Rahim, and K. Ali, "Families of regular graphs with constant metric dimension," Utilitas Mathematica, vol. 75, pp. 21-33, 2008.

[11] I. Tomescu and I. Javaid, "On the metric dimension of the Jahangir graph," Bulletin Mathématique de la Société des Sciences Mathématiques de Roumanie, vol. 50, no. 4, pp. 371-376, 2007.

[12] M. Imran, A. Q. Baig, M. K. Shafiq, and I. Tomescu, "On metric dimension of generalized Petersen graphs $P(n ; 3)$," ARS Combinatoria, vol. 117, pp. 113-130, 2014.

[13] J.-B. Liu, C. Wang, S. Wang, and B. Wei, "Zagreb indices and multiplicative zagreb indices of eulerian graphs," Bulletin of the Malaysian Mathematical Sciences Society, vol. 42, no. 1, pp. 67-78, 2019.

[14] J. B. Liu, J. Zhao, J. Min, and J. Cao, "The Hosoya index of graphs formed by a fractal graph,” Fractals, vol. 27, no. 8, Article ID 1950135, 2019.

[15] J.-B. Liu and S. N. Daoud, "Number of spanning trees in the sequence of some graphs," Complexity, vol. 2019, Article ID 4271783, 22 pages, 2019.

[16] J. B. Liu, J. Zhao, and Z. Q. Cai, "On the generalized adjacency, Laplacian and signless Laplacian spectra of the weighted edge corona networks," Physica A: Statistical Mechanics and Its Applications, vol. 540, 2020.

[17] J. B. Liu, Z. Y. Shi, Y. H. Pan, J. Cao, M. Abdel-Aty, and U. Al-Juboori, "Computing the laplacian spectrum of linear octagonal-quadrilateral networks and its applications," Polycyclic Aromatic Compounds, pp. 1-2, 2020.

[18] J. B. Liu, X. F. Pan, J. Cao, and F. F. Hu, "A note on some physical and chemical indices of clique-inserted lattices," Journal of Statistical Mechanics: Theory and Experiment, vol. 2014, no. 6, Article ID P06006, 2014.

[19] J.-B. Liu, X.-F. Pan, J. Cao, and X. Huang, "The kirchhoff index of toroidal meshes and variant networks," Mathematical Problems in Engineering, vol. 2014, Article ID 286876, 8 pages, 2014.

[20] J.-B. Liu, X.-F. Pan, and J. Cao, "Some properties on Estrada index of folded hypercubes networks," Abstract and Applied Analysis, vol. 2014, Article ID 167623, 6 pages, 2014. 
[21] J.-B. Liu, J. Cao, and J. Xie, "On the incidence energy of some toroidal lattices," Abstract and Applied Analysis, vol. 2014, Article ID 568153, 6 pages, 2014.

[22] J. Currie and O. R. Oellermann, "The metric dimension and metric independence of a graph," Journal of Combinatorial Mathematics and Combinatorial Computing, vol. 39, pp. 157-167, 2001.

[23] M. Fehr, S. Gosselin, and O. R. Oellermann, "The metric dimension of Cayley digraphs," Discrete Mathematics, vol. 306, no. 1, pp. 31-41, 2006.

[24] M. Feng, B. Lv, and K. Wang, "On the fractional metric dimension of graphs," Discrete Applied Mathematics, vol. 170, pp. 55-63, 2014.

[25] M. Feng and K. Wang, "On the metric dimension and fractional metric dimension for hierarchical product of graphs," Applicable Analysis and Discrete Mathematics, vol. 7, no. 2, pp. 302-313, 2013.

[26] J.-B. Liu, A. Kashif, T. Rashid, and M. Javaid, "Fractional metric dimension of generalized Jahangir graph," Mathematics, vol. 7, no. 1, p. 100, 2019.

[27] J.-B. Liu, M. K. Aslam, and M. Javaid, "Local fractional metric dimensions of rotationally symmetric and planar networks," IEEE Access, vol. 8, no. 1, pp. 82404-82420, 2020.

[28] S. Aisyah, M. I. Utoyo, and L. Susilowati, "On the local fractional metric dimension of corona product graphs," IOP Conference Series Earth and Environmental Science, vol. 243, no. 1, Article ID 012043, 2019.

[29] S. W. Saputro, A. Semanicova-Fenovc, M. Baca, and M. Lascsakova, "On fractional metric dimension of comb product graphs," Statistics Optimization \& Information Computing, vol. 6, no. 1, pp. 150-158, 2018.

[30] E. Yi, "The fractional metric dimension of permutation graphs," Acta Mathematica Sinica, English Series, vol. 31, no. 3, pp. 367-382, 2015.

[31] R. A. Melter and I. Tomescu, "Metric bases in digital geometry," Computer Vision, Graphics, and Image Processing, vol. 25, no. 1, pp. 113-121, 1984.

[32] A. Shahbaz and M. Khalid, "A paradigmatic approach to investigate restricted totient graphs and their indices," International Journal of Mathematics and Computer Science, vol. 16, no. 2, pp. 793-801, 2021.

[33] S. Ali, M. K. Mahmood, and M. H. Mateen, "New labeling algorithm on various classes of graphs with applications," in Proceedings of the 2019 International Conference on Innovative Computing (ICIC), pp. 1-6, Lahore, Pakistan, November 2019.

[34] M. Khalid and A. Shahbaz, "A novel labeling algorithm on several classes of graphs," Punjab University Journal of Mathematics, vol. 49, pp. 23-35, 2017.

[35] A. Shahbaz and M. Khalid, "New numbers on Euler's totient function with application," Journal of Mathematical Extension, vol. 14, pp. 61-83, 2020.

[36] M. Khalid and A. Shahbaz, "On super totient numbers with applications and algorithms to graph labeling," Ars Combina, vol. 143, pp. 29-37, 2019.

[37] M. Javaid, M. Raza, P. Kumam, and J.-B. Liu, "Sharp bounds of local fractional metric dimensions of connected networks," IEEE Access, vol. 8, no. 1, pp. 172329-172342, 2020. 